The Astrophysical Journal, 635:311-335, 2005 December 10

(C) 2005. The American Astronomical Society. All rights reserved. Printed in U.S.A.

\title{
BLUE LUMINOUS STARS IN NEARBY GALAXIES: QUANTITATIVE SPECTRAL ANALYSIS OF M33 B-TYPE SUPERGIANT STARS ${ }^{1,2}$
}

\author{
M. A. URbaneja \\ Instituto de Astrofísica de Canarias, Vía Láctea S/N, E-38205 La Laguna, Canary Islands, Spain; and Institute for Astronomy, \\ University of Hawaii, 2680 Woodlawn Drive, Honolulu, HI 96822; urbaneja@ifa.hawaii.edu \\ A. HERrero \\ Instituto de Astrofísica de Canarias, Vía Láctea S/N, E-38205 La Laguna, Canary Islands, Spain; and Departamento de Astrofísica, \\ Universidad de La Laguna, Avda. Astrofísico Francisco Sánchez, E-38271 La Laguna, Canary Islands, Spain; ahd@iac.es
}

R.-P. KUDRITZKI

Institute for Astronomy, University of Hawaii, 2680 Woodlawn Drive, Honolulu, HI 96822; kud@ifa.hawaii.edu

F. NAJARRo

Instituto de Estructura de la Materia, CSIC, C/Serrano, 121, E-28006 Madrid, Spain; najarro@isis.iem.csic.es

S. J. SMARTT

Department of Physics and Astronomy, Queen’s University Belfast, Belfast BT7 1NN, UK; s.smartt@qub.ac.uk

J. Puls

Universitäts-Sternwarte München, Scheinerstrasse 1, D-81679 Munich, Germany; uh101aw@usm.uni-muenchen.de

D. J. LENNON

Isaac Newton Group of Telescopes, Apartado 321, 38700 Santa Cruz de La Palma, Canary Islands, Spain; dj1@ing.iac.es

AND

L. J. Corral

Instituto de Astrofísica de Canarias, Vía Láctea S/N, E-38205 La Laguna, Canary Islands, Spain; and GRANTECAN, Vía Láctea S/N, E-38205 La Laguna, Canary Islands, Spain; lcorral@iac.es

Received 2005 February 6; accepted 2005 August 26

\begin{abstract}
We present the detailed spectral analysis of a sample of M33 B-type supergiant stars, aimed at the determination of their fundamental parameters and chemical composition. The analysis is based on a grid of non-LTE metal lineblanketed model atmospheres including the effects of stellar winds and spherical extension computed with the code FASTWIND. Surface abundance ratios of $\mathrm{C}, \mathrm{N}$, and $\mathrm{O}$ are used to discuss the chemical evolutionary status of each individual star. The comparison of observed stellar properties with theoretical predictions of massive star evolutionary models shows good agreement within the uncertainties of the analysis. The spatial distribution of the sample allows us to investigate the existence of radial abundance gradients in the disk of M33. The comparison of stellar and $\mathrm{H}$ II region $\mathrm{O}$ abundances (based on direct determinations of the electron temperature of the nebulae) shows good agreement. Using a simple linear radial representation, the stellar oxygen abundances result in a gradient of $-0.0145 \pm 0.005 \mathrm{dex} \operatorname{arcmin}^{-1}$ (or $-0.06 \pm 0.02 \mathrm{dex} \mathrm{kpc}^{-1}$ ) up to a distance equal to $\sim 1.1$ times the isophotal radius of the galaxy. A more complex representation cannot be completely discarded by our stellar sample. The stellar $\mathrm{Mg}$ and $\mathrm{Si}$ abundances follow the trend displayed by $\mathrm{O}$ abundances, although with shallower gradients. These differences in gradient slope cannot be explained at this point. The derived abundances of the three $\alpha$-elements yield solar metallicity in the central regions of the disk of M33. A comparison with recent planetary nebula data from Magrini and coworkers indicates that the disk of M33 has not suffered from a significant $\mathrm{O}$ enrichment in the last 3 Gyr.
\end{abstract}

Subject headings: galaxies: abundances — galaxies: individual (M33, NGC 598) — galaxies: ISM — stars: abundances — stars: atmospheres — stars: fundamental parameters

\section{INTRODUCTION}

Understanding massive hot luminous stars is of fundamental importance to our knowledge of the universe. Massive hot stars play a key role in the chemodynamical evolution of galaxies, by

\footnotetext{
${ }^{1}$ The WHT is operated on the island of La Palma by the ING in the Spanish Observatorio del Roque de los Muchachos of the Instituto de Astrofísica de Canarias.

${ }^{2}$ Some of the data presented herein were obtained at the W. M. Keck Observatory, which is operated as a scientific partnership among the California Institute of Technology, the University of California, and the National Aeronautics and Space Administration. The Observatory was made possible by the generous financial support of the W. M. Keck Foundation.
}

injecting momentum and kinetic energy into the interstellar medium (ISM), by returning material, both pristine and nuclear processed, during their whole lifetime via the stellar winds, and, finally, in their spectacular death as supernovae. With masses above 8 times the mass of the Sun, blue supergiants gain energy from rather efficient thermonuclear reactions (the carbon-nitrogenoxygen cycle), which consume the gas reservoir in a short period of time. Typically, the main-sequence (MS) lifetime of a hot massive star lasts for several million years, much shorter than for a solar-like star. The huge amount of energy released in their cores produces dramatic effects on the star and on the surrounding medium, triggering strong radiation-driven winds (Kudritzki 
\& Puls 2000 and references therein) that steadily strip off the star.

Even with the advances accomplished in recent years, the evolution of massive stars in the upper regions of the HertzsprungRussel diagram (HRD) is not yet entirely understood. Evolutionary models still contain uncertainties that can strongly affect the evolutionary path of a given star in the HRD, even during the MS. This becomes particularly true for A- and B-type supergiants beyond the MS, as these uncertainties accumulate during the evolution to the red giant branch (RGB). Whether massive stars perform blue loops or not after they reached the RGB is still an open question. If they do, then the blue supergiant population must be a mixture of pre- and post-red supergiant phase stars, which must be reflected in a wide spread of their masses and surface chemical compositions. The analyses of massive blue stars and related objects carried out to date (among others, we cite here the works by Lennon et al. 1991; Venn 1995, 1999; McErlean et al. 1999; Korn et al. 2000; Lamers et al. 2001; Venn et al. 2002; Smartt et al. 2002; Villamariz et al. 2002; Przybilla 2002; Lennon et al. 2003; Trundle et al. 2004) are consistent with the existence of a mixing mechanism of chemical elements that operates already at early stages of massive stellar evolution, even during the MS. In recent work, stellar rotation is proposed as the cause of this mixing (Heger et al. 2000; Heger \& Langer 2000; Maeder \& Meynet 2001). The question of blue loops and additional mixing provided by them is still unresolved.

With an investigation of massive stars in spiral galaxies such as M33 and M31 one can address a number of important issues. Studies of massive stars in our own Galaxy are usually hampered by uncertainties in the distance, which translate into large uncertainties of the stellar parameters. Therefore, stars in external galaxies, where the relative errors in distance are smaller, are ideal to test evolutionary scenarios. In addition, studies of surface chemical composition of early-type stars can also help in our understanding of the galaxies themselves because they allow us to constrain chemical abundance gradients, which constitute one of the most important observational constraints for models of galactic chemical evolution (Stasińska 2004). Among the normal stellar population of a galaxy, B- and A-type supergiants are ideal objects to trace the spatial distribution of chemical elements. They have an enormous intrinsic brightness and their blue spectra are rich in metal absorption lines $(\mathrm{C}, \mathrm{N}, \mathrm{O}, \mathrm{Ne}, \mathrm{Mg}, \mathrm{Al}$, $\mathrm{Si}, \mathrm{S}, \mathrm{Ti}, \mathrm{Fe}, \ldots .$.$) . This renders the possibility of chemical$ abundance studies in galaxies beyond the Magellanic Clouds (McCarthy et al. 1995, 1997; Monteverde et al. 1997, 2000; Venn 1999; Venn et al. 2000, 2003; Smartt et al. 2001a; Trundle et al. 2002; Kaufer et al. 2004) and even beyond the Local Group (Bresolin et al. 2001, 2002; Urbaneja et al. 2003, 2005). Moreover, blue supergiant stars offer two additional advantages. First, they can be found in the outer regions of galactic disks, where the $\mathrm{H}$ II regions are fainter and more difficult to observe, and can be used as tracers to explore the proposed flattening of the abundance gradients in the outer disk of galaxies (Fich \& Silkey 1991; Vílchez \& Esteban 1996; Rudolph et al. 1997; Deharveng et al. 2000; Pilyugin 2003). Second, the analysis of blue supergiant stars located close to the galactic centers can help to resolve a systematic uncertainty of ISM O/H abundance analysis methods at high metallicity (above $\sim 0.5 Z_{\odot}$ ). Recent studies of (possibly) high-metallicity $\mathrm{H}$ II regions in external galaxies (M101, Kennicutt et al. 2003; M51, Garnett et al. 2004; Bresolin et al. 2004) detected large systematic differences when comparing oxygen abundances obtained from a direct determination of the electron temperature $T_{e}$ of the nebulae via auroral lines and abundances derived by using widely accepted calibrations of sta- tistical methods (the so-called strong line methods). Abundances derived with the direct method seem to be lower by a factor of 2-3. On the other hand, Stasińska $(2002,2005)$ found that $T_{e}$-based abundances underestimate the "true abundances" for $\log (\mathrm{O} / \mathrm{H})+$ $12>8.7$ and never reach values above the solar abundance. Another complication arises from the possible existence of $T_{e}$ fluctuations (Peimbert 1967), which might be responsible for the large abundance discrepancies found between recombination and collisionally excited lines of the same ionic species within a nebula (see, for example, the recent reinvestigation of the chemical content of the Orion Nebula by Esteban et al. 2004).

An investigation of these problems is relevant for not only element abundance gradients in nearby galaxies but also the study of the chemical content of galaxies at different redshifts, i.e., the chemical evolution history of the universe. An independent method to determine the oxygen abundances at possibly high metallicity as through the analysis of blue supergiants offers an important alternative to assess the accuracy of nebular emission line abundance studies.

Within the Local Group of galaxies, the late-type spiral galaxy M33 represents the best laboratory for studies of its blue supergiant stars because of its close proximity $(\mu=24.56 \pm$ 0.10 ; Freedman et al. 2001), its nearly face-on geometry (translating into a low internal reddening and extinction), and its large oxygen (and silicon) radial abundance gradient (Monteverde et al. 2000). Because of these gradients, M33 offers the possibility to study blue supergiants (their general properties and their evolution) in a wide range of metallicity at a (fairly) common distance. The high-luminosity tail of the blue supergiant population is expected to have visual apparent magnitudes above $19 \mathrm{mag}$, still achievable for a medium-aperture class telescope. The goals of this work are the determination of the surface chemical composition (i.e., the chemical evolutionary stage) of a large sample of B-type supergiant stars, the determination of the stellar $\alpha$-element radial abundance gradients over a substantial fraction of the disk of M33, and the comparison of these stellar abundances with the ISM abundances obtained by direct methods (Vílchez et al. 1988). In a forthcoming paper we will discuss the wind momentum-luminosity relationship of the stars presented here along with a larger sample of M33 blue supergiants.

In the following sections we present the stellar spectra to be analyzed $(\S 2)$, together with a discussion of the analysis method and the model atmospheres $(\S 3)$. In $\S 4$ we compare our results with chemical abundances obtained from other investigations. Finally, we present the conclusions of this study in $\S 5$, along with an outline of the ongoing work.

\section{OBSERVATIONS AND DATA REDUCTION}

The optical spectra of nearly 30 blue supergiants have been collected during the past 10 years using different telescopes and instruments. The observational project started in 1994, using the 4.2 m class William Herschel Telescope (WHT) equipped with the Intermediate Spectrograph and Imaging System (ISIS), a double-armed medium-resolution optical spectrograph. A complete description of the early observations, between 1994 and 1997, can be found in Herrero et al. (1994) and Monteverde et al. $(1996,1997)$. The spectral resolution of these early observations is $R=2500$ (or $\sim 2 \AA$, FWHM, in the final extracted spectra) with a signal-to-noise ratio $(\mathrm{S} / \mathrm{N})$ in the continuum close to 60 , except for the "most luminous" objects, 110A and 1054 (see the individual comments in $\S 3.5 .1$ ). The star B38, presented in Monteverde et al. (2000), was observed at an improved resolution of $R=5000$ ( $1 \AA$ FWHM $)$. 
TABLE 1

Observation Logbook of M33 Supergiant Stars

\begin{tabular}{|c|c|c|c|c|c|c|c|}
\hline ID & $\begin{array}{c}\text { R.A. } \\
(\mathrm{J} 2000.0)\end{array}$ & $\begin{array}{c}\text { Decl. } \\
(\mathrm{J} 2000.0)\end{array}$ & $\begin{array}{c}m_{V} \\
(\mathrm{mag})\end{array}$ & Spectral Class & $\begin{array}{c}\mathrm{S} / \mathrm{N} \\
(4200 / 4580)\end{array}$ & $R$ & References \\
\hline $0900 \ldots \ldots$ & 013344.90 & +303616.70 & 17.30 & B0.5-B1 Ia & $40-50$ & 2500 & 1 \\
\hline 110A & 013341.00 & +302237.00 & 16.10 & $\mathrm{~B} 1 \mathrm{Ia}^{+}$ & $100-78$ & 2500 & 2 \\
\hline В38 & 013300.83 & +303505.10 & 17.30 & B1 Ia & $55-65$ & 5000 & 3 \\
\hline В133 & 013329.00 & +304744.00 & 16.69 & B1.5 Ia & $70-55$ & 2500 & 2 \\
\hline $1054 \ldots \ldots \ldots \ldots \ldots$ & 013350.83 & +303834.50 & 16.49 & B2 Ia & $100-120$ & 2500 & 2 \\
\hline $1137 \ldots \ldots \ldots$ & 013353.23 & +303526.10 & 17.07 & $\mathrm{~B} 2-3 \mathrm{Ib}$ & $60-70$ & 2500 & 1 \\
\hline OB $10-3 \ldots \ldots$ & 013335.73 & +310047.00 & 18.20 & O8:If & $60-67$ & 5000 & 4 \\
\hline OB $112-41 \ldots \ldots \ldots \ldots \ldots$ & 013342.02 & +302142.30 & 18.01 & B0 Ia & $70-65$ & 5000 & 4 \\
\hline UIT $103 \ldots \ldots \ldots \ldots \ldots \ldots \ldots$ & 013327.29 & +310056.70 & 17.88 & B0.5-B0.7 Ia & $80-80$ & 5000 & 1 \\
\hline UIT $122 \ldots \ldots \ldots \ldots \ldots \ldots \ldots$ & 013333.70 & +304720.20 & 17.81 & B0.5 Ia & $100-80$ & 5000 & 1,5 \\
\hline UIT $136 \ldots \ldots \ldots \ldots . . . . . . . . . . .$. & 013335.73 & +310047.00 & 17.98 & B0.5 Ia & $88-100$ & 5000 & 1 \\
\hline OB $10-10$ & 013344.22 & +303148.20 & 17.30 & $\mathrm{~B} 1 \mathrm{Ia}^{+}$ & $87-80$ & 5000 & 4 \\
\hline
\end{tabular}

Notes.-Units of right ascension are hours, minutes, and seconds, and units of declination are degrees, arcminutes, and arcseconds. The first group has been observed with WHT ISIS, while the second one has been observed using Keck ESI.

References.-(1) This paper; (2) Monteverde et al. 1996; (3) Monteverde et al. 2000; (4) Massey et al. 1995; (5) Massey et al. 1996.

In addition, 14 new blue targets were observed with WHT ISIS in 2000 October during five nights, split into two groups of three and two nights (October 6, 7, and 8, and October 22 and 23, respectively). Aimed at avoiding unresolved groups of stars that could appear as bright isolated supergiants (see the discussion for B133), the new targets were selected among the objects with visual magnitude around 18th, close to the magnitude limit of this observational setup, in combination with Hubble Space Telescope (HST) images (in those cases in which these were available). Although ISIS allows for the simultaneous acquisition of blue and red spectrograms by means of a dichroic beam splitter, our previous experience has shown that the use of this device introduces some structure into the blue spectra, which is difficult to completely remove from the extracted spectra. It also reduces the throughput of the blue arm and compromises the S/N. Consequently, we used only the blue arm. These observations cover the 3950-4700 $\AA$ wavelength range at $R=5000$ with an $\mathrm{S} / \mathrm{N}$ in the continuum around 60 .

Four of the confirmed isolated blue supergiants, along with two new targets, were observed one year later, on October 21 and 22, using ESI (Sheinis et al. 2002) at the Keck II telescope. These observations cover a wider spectral range, from almost the atmospheric cutoff in the blue to $1.0 \mu \mathrm{m}$, at $R=5000$. Due to cosmetic defects in the CCD detector of ESI, the key lines Mg II $\lambda 4481$ and $\mathrm{He}$ I $\lambda 4471$ were lost. Fortunately, we could recover these lines from the previous WHT ISIS observations.

A summary of the photometric magnitudes, along with some other relevant information concerning the selected M33 OB supergiants to be analyzed, is compiled in Table 1 .

Wavelength-calibrated spectra were obtained from the processed CCD frames using standard procedures implemented within the IRAF reduction system. ${ }^{3}$ The final steps toward the extracted spectra were performed using our own specific routines developed within the IDL software. Our analysis method does not require a flux calibration. It is sufficient to work with normalized spectra. The rectification was done with a procedure that uses pure continuum spectral windows, i.e., a number of small wavelength ranges that are free of lines. We have extended the windows defined by Lennon et al. (1992), up to $6000 \AA$ by a

\footnotetext{
${ }^{3}$ IRAF is written and supported by the IRAF programming group at the National Optical Astronomy Observatory (NOAO) in Tucson (http://iraf.noao
} .edu). careful inspection of high-S/N (above 350 in the continuum) and high-resolution $(R=15,000)$ echelle spectra of Galactic B-type supergiants obtained with the $2.1 \mathrm{~m}$ telescope in the San Pedro Mártir Observatory, Baja California, Mexico. Previous to the application of the window procedure, the spectra have to be corrected for the radial velocities. We cross-correlate the observed spectra (after a preliminary rectification) with a set of generic synthetic spectra. We mask the $\mathrm{H}$ and $\mathrm{He}$ lines for the correlation, in order to avoid any possible contamination due to a shift on their rest wavelength produced by the stellar wind outflow. Once the radial velocity is known, the spectrum can be finally normalized. The final spectra are displayed in Figures 1, 2, and 3 (WHT ISIS observations, Keck ESI blue data, and stars observed with WHT ISIS, not finally considered in the analysis presented in this paper, respectively; see below).

The projected rotational velocities, $v \sin i$, were determined from the extracted spectra using a standard procedure comparing profiles of observed metal lines with calculated lines convolved to different rotational velocities. This procedure assumes that the broadening of the line, after removing the instrumental profile (adopted to be Gaussian), is produced entirely by the stellar rotation. We are aware of the work by Ryans et al. (2002) suggesting that the rotational velocities derived to date using this standard procedure overestimate the true values because of the fact that a significant fraction of the broadening can actually be the result of the presence of large-scale (macroturbulent) motions in the atmosphere. It is still unclear whether or not both broadening mechanisms, rotation and macroturbulence, can be clearly distinguished. In particular, the nature of the mechanism that could produce the macroturbulence remains elusive (convection, instabilities in driving the mass outflows, nonradial oscillations, etc.). At the resolution and $\mathrm{S} / \mathrm{N}$ of our spectra it is not possible to disentangle the contribution from a broadening process other than the classic stellar rotation. However, our analysis is not affected by this shortcoming since the strengths of the absorption-line features conserved under both broadening mechanisms, rotation and macroturbulence, and the resulting theoretical profile differences are very small. The abundance analysis and the determination of stellar parameters are, therefore, not affected.

\subsection{Spectral Classification}

Previous to a detailed spectral analysis on the basis of model atmospheres, the spectral classification of the stars provides 


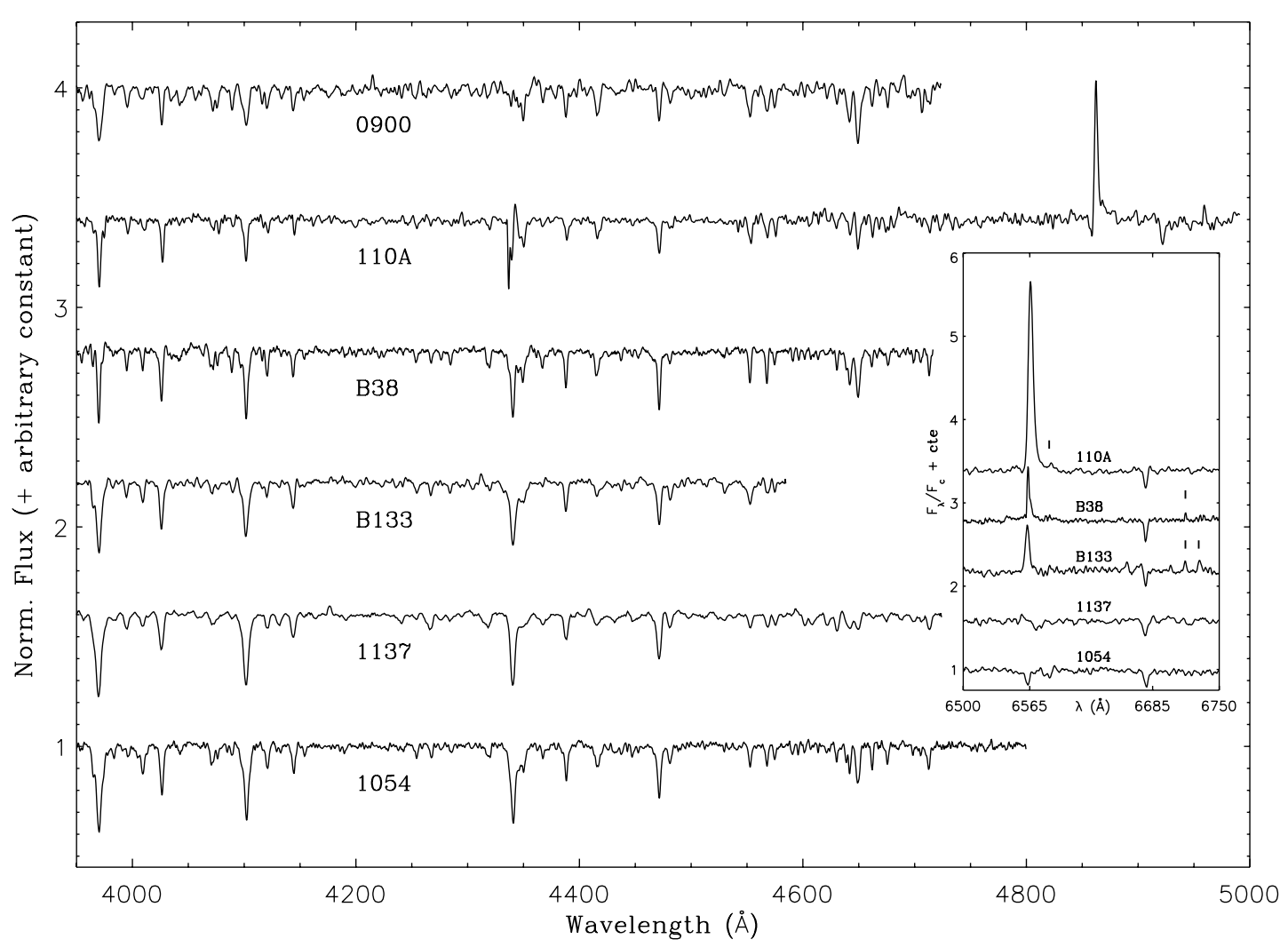

FIG. 1.-Spectra of M33 B-type supergiants obtained with WHT ISIS. The normalized spectra have been shifted arbitrarily in the $y$-axis for the sake of clarity. The inset shows the $\mathrm{H} \alpha$ spectra, where we have marked IS emission lines by short thick vertical lines.

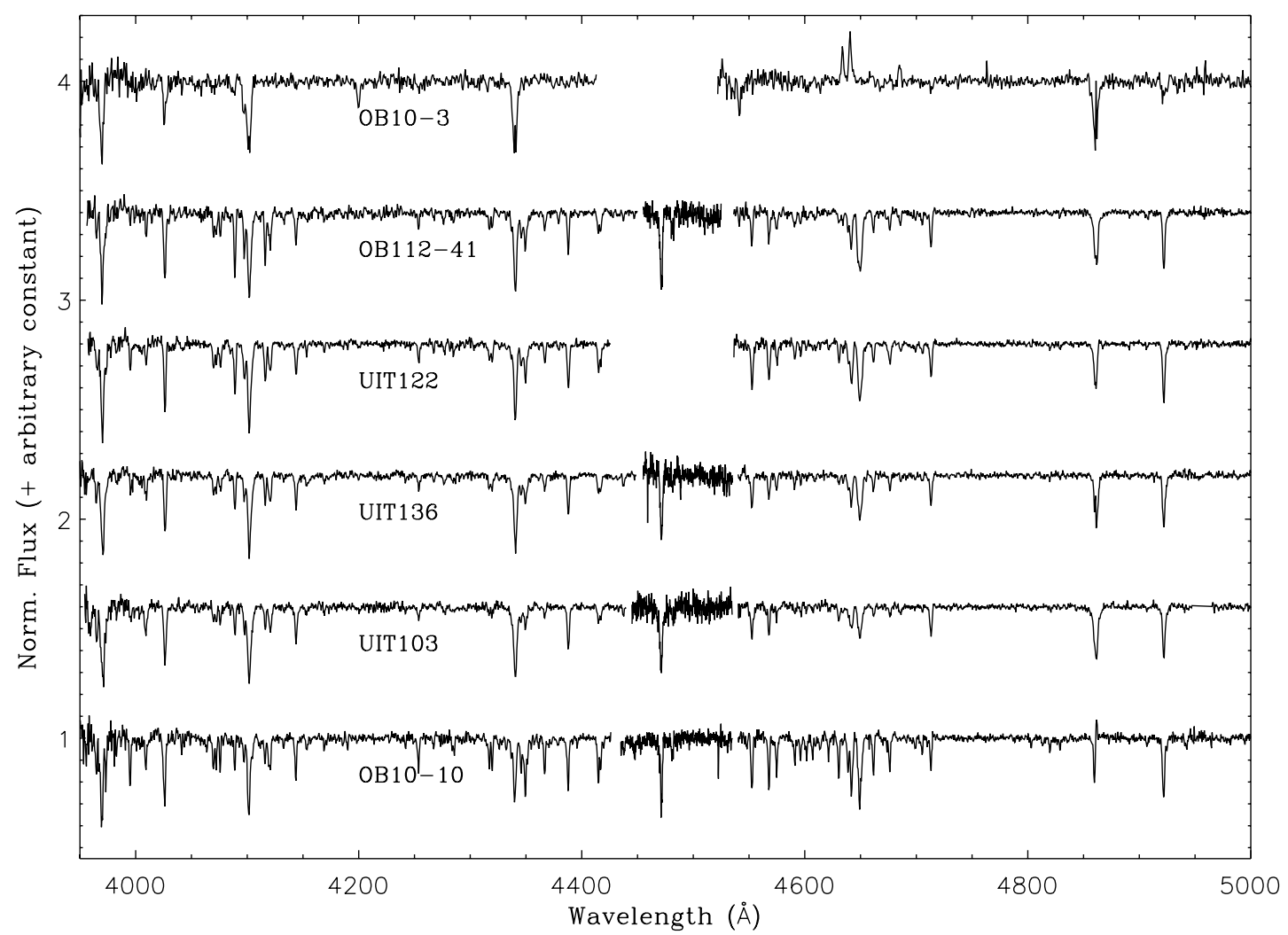

FIG. 2.-Blue Keck ESI spectra. Note that the $4450-4540$ Å regions were lost in these data due to cosmetic problems of the CCD. We have included the WHT ISIS spectra of this regions when available. As in the previous figure, the spectra have been shifted in the $y$-axis for clarity. 


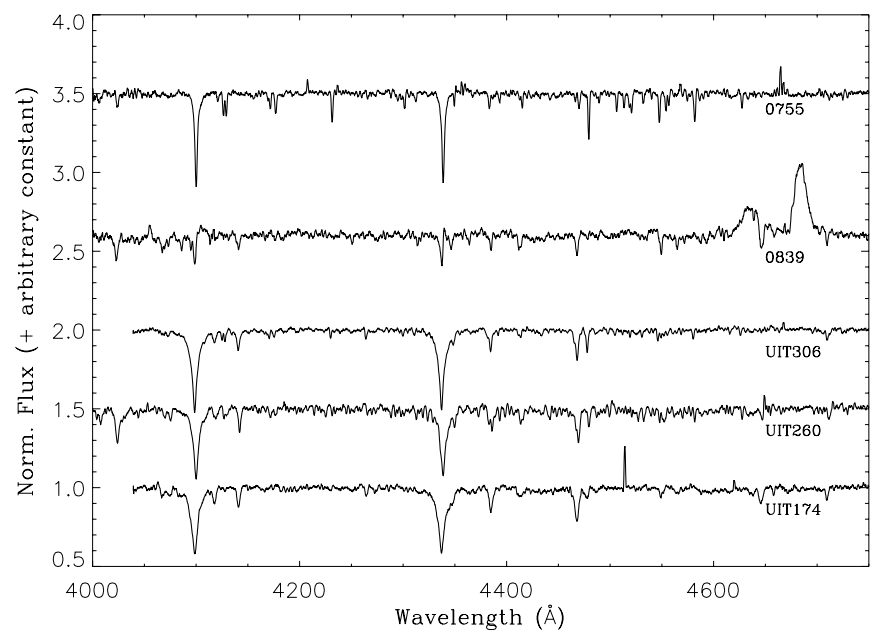

FIG. 3.-WHT ISIS blue spectra of several M33 not included in the analyses. Top to bottom: 0755 (A1-A2 Ia), 0839 (WN7+absorption), UIT 306, and the composite spectra of UIT 260 and UIT 174 (see text for comments).

helpful information to restrict the possible range of stellar parameters. Although many of the stars that are analyzed here have been previously classified elsewhere (see references in Table 1), these classifications must be checked because they were generally based on photometric surveys or, in the best cases, on lowresolution spectroscopy.

Unlike O-type stars (and the earlier B type, B0), which are classified on the basis of their $\mathrm{He}$ I and $\mathrm{He}$ II lines, B- and A-type stars are classified by comparing the relative strength of metal to He I lines, particularly from the relative strengths of silicon and magnesium lines (Walborn \& Fitzpatrick 1990; Lennon et al. 1992). An important issue concerning this classification scheme that has to be considered when dealing with extragalactic stellar spectra is that stars can have very different metallicities. This is true for stars in M33, which has steep metallicity gradients (VilaCostas \& Edmunds 1992; Garnett et al. 1997; Monteverde et al. 2000). As has been discussed by Lennon (1997), different metallicities can mimic the effects of different spectral types. To circumvent this problem, it is important to use stellar templates of different metallicities (Monteverde et al. 1996; Bresolin et al. 2002). Another important point to be considered is the dependence of the strength of many metal lines on luminosity class. As a direct consequence, spectral classifications cannot rely on metal lines only. As argued by Monteverde et al. (1996), the consideration of the hydrogen Balmer lines, along with the metal features, allows for the simultaneous spectral type and luminosity classification, since $\mathrm{H}$ Balmer lines react strongly to luminosity, especially $\mathrm{H} \alpha$. The sole use of metal lines to disentangle the luminosity class can lead to seriously inconsistent classifications (the reader is referred to Lennon [1997] for a detailed discussion).

We apply the following procedure. First, we assume that all of the observed stars have the same metallicity and compare our spectra with Galactic B-type supergiants with well-established spectral classifications. This allows us to define a first rough division in several spectral bins. In a second step, we compare the observed spectra with low-metallicity templates, using SMC B-type supergiants from Lennon (1997). Our former classification is then refined by considering the effects of the different metallicities. An additional advantage of this general procedure of classification is that it also provides us with a first estimate of metallicity, as well as of the relative strength of their mass outflow, from the comparisons of $\mathrm{H} \alpha$ profiles.
In the following we discuss only the spectral classification of those stars for which we found discrepancies with previous respective classifications.

Object 0900, aka B217a, is the star with the lowest quality spectrum, showing an S/N of 40-50 only. There are no signs of He II lines, except perhaps for He II $\lambda 4686$ (see Fig. 1); however, strong $\mathrm{Si}$ IV lines are present without any evidence of Si II lines. Also, the Si III $\lambda \lambda 4552,4567,4574$ lines are stronger than the $\mathrm{Mg}$ II $\lambda 4481$ line. All of these characteristics, along with the strength of the metal features around $4650 \AA$, point to a type earlier than B1.5. From comparisons with Galactic B0.5 Ia and $\mathrm{B} 1 \mathrm{Iab}$ stars and considering the poor quality, we conclude that 0900 is near Galactic metallicity and shows the spectrum of a B0-B1 Ia star. This classification is consistent with the UV spectrum discussed in Urbaneja et al. (2002); the top panel of Figure 4 displays the comparison of the UV spectra of 0900 and the B0.5Ia star UIT 122 (see below), confirming our conclusion (the spectrum of UIT 122 has been smoothed because of its low $\mathrm{S} / \mathrm{N}$ ).

The star 1137, alias B354 and UIT 235, was classified by Massey et al. (1996) as B1 III: based on photometric colors. However, this classification is not consistent with the observed $\mathrm{H}$ Balmer lines, which are narrow and indicate a higher luminosity class. Along with that, 1137 shows weak indications of Si IV lines. The identification of the strong feature around $4130 \AA$ as an $\mathrm{Si}$ II line is somewhat doubtful because of the lack of the other doublet companion at $4128 \AA$. This strong feature, on the other hand, could be the resulting blend of two $\mathrm{O}$ II lines, which will require the combination of both an earlier type than $\mathrm{B} 2$ and high metallicity. However, other strong $\mathrm{O}$ II lines would have to be present in the spectrum (for example, the group of lines at $\sim 4060$ $4080 \AA$ ), and this is not the case; we cannot provide a solid identification for this feature currently. The rest of the observed spectrum is compatible with a type cooler than B1 with a high luminosity class and a metallicity somewhat lower than the Galactic references. We tentatively classify 1137 as a B2-B2.5 Ib.

Finally, we discuss UIT 136 and UIT 103, both classified as generic OB types in Massey et al. (1996). For UIT 136 we choose the same classification as for UIT 122, B0.5 Ia (Massey et al. 1996), because of the close resemblance of their optical spectra (see Fig. 2). In the case of UIT 103, the star could be slightly cooler than UIT 122, around a B0.7 Ia type, but differences might also be just an effect of a lower metallicity. Thus, we classify it as a B0.5-B0.7 Ia star.

\subsubsection{Discarded Spectra}

A few objects turned out not to be useful for the purposes of this work. Here we present a short discussion of their spectra, which are displayed in Figure 3. In the following, all of the referred broadband images belong to the survey by Massey et al. (2001).

UIT 174 was classified as a B1 I by Massey et al. (1996). This star displays side extensions in the east-west line in $U, B$, and $I$ images. The intensity profile of our spectrum perpendicular to the direction of dispersion confirms the presence of another object, a conclusion that is also supported by the broad Balmer lines.

UIT 260, or OB 5-8, also has a close companion object in $B, V$, and $I$ images, where the star shows an elongated southeast appearance. The extracted spectrum displays broad Balmer lines as a result of this contamination.

UIT 306 has been classified as a B3 Ia by Massey et al. (1996). Its blue spectrum shows broad Balmer lines, which indicates a higher luminosity class. The star looks isolated in $U, V$, and $I$ images. However, given the visual magnitude reported by Massey et al. (1996), $m_{V}=16.53 \mathrm{mag}$, the distance to M33, and assuming a mean reddening of $0.13 \mathrm{mag}$, the absolute $V$ magnitude 

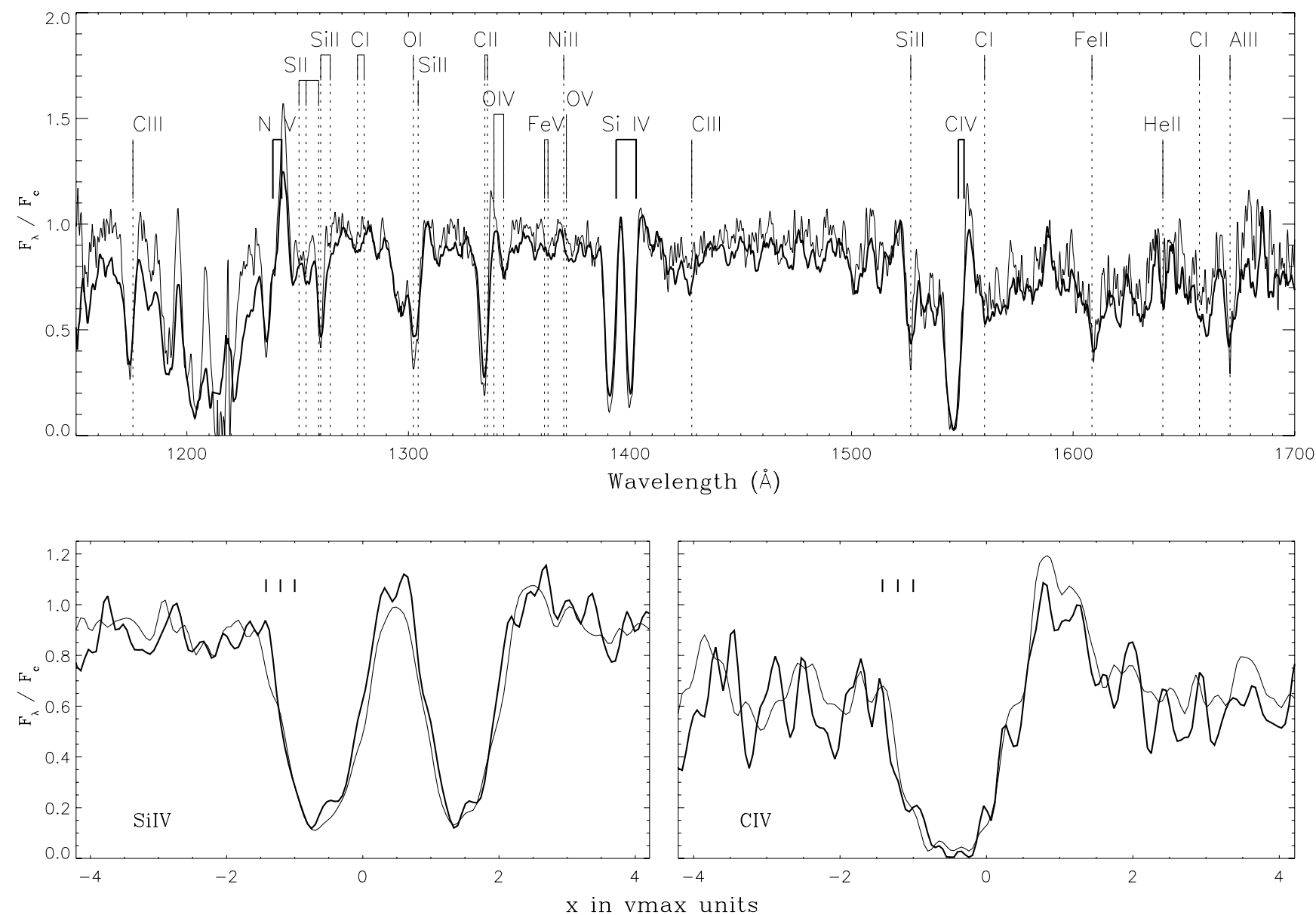

FIG. 4.-Comparison of HST STIS UV spectra of 0900 (thin line) and UIT 122 (thick line; HST archive image O6DJ09020, GO program 9127, PI: Bianchi). In the top panel, the main ISM lines are identified at the top, and below we also show the rest wavelengths of some important stellar lines. In the bottom panels, the three short vertical lines display the location of $v_{\infty}$ of 0900 , as well as a $10 \%$ variation of this value.

inferred, $-8.4 \mathrm{mag}$, corresponds to a supergiant star. A possible interpretation, considering both the broad Balmer lines and the high apparent visual magnitude, is that this object could be indeed a compact group of stars. Another possible explanation that cannot be completely ruled out at present is that the star is a foreground Galactic object.

Object 0755 (Ivanov et al. 1993) was included in the slit during the WHT ISIS observation of OB 112-41 (the stars are separated by about $8^{\prime \prime}$ ). From the gathered blue spectrum, we classify this star as an A1-A2 supergiant. A complete detailed analysis will be performed after new observations are available, covering a much larger wavelength range.

Finally, the blue spectrum of 0839 (Ivanov et al. 1993), also known as UIT 176 and WR 91-129 (Massey \& Johnson 1998), is characteristic of a WN Wolf-Rayet star. The N v $\lambda \lambda 4603,4619$ lines are absent. The $\mathrm{N}$ III $\lambda \lambda 4634-4641$ emission is weaker than the He II $\lambda 4686$ feature but stronger than the $\mathrm{N}$ iv $\lambda 4058$ feature. Along with these $\mathrm{WN}$ features, the spectrum displays strong absorptions at the location of the $\mathrm{H}$ lines. Our spectrum confirms then the previous classification as a WN7+abs given by Massey et al. (1996).

\section{SPECTRAL ANALYSIS}

The basis of our analysis method is the comparison of the observed spectra with the calculations from a large grid of nonLTE metal line blanketed model atmospheres including the hydrodynamic effects of stellar winds and the geometrical effects of spherical extension computed with the code FASTWIND (Santolaya-Rey et al. 1997; Puls et al. 2003). Here we describe very briefly the code and the model atoms and discuss the methodology and its limitations in some detail.

\subsection{Model Atmospheres and Model Atoms}

\subsubsection{Model Atmosphere/Line Synthesis Code}

FASTWIND solves the radiative transfer equation in the comoving frame of the expanding atmospheres of early-type stars in extended spherical geometry, subject to the constraint of statistical equilibrium. Steady state is assumed, as well as a homogeneous composition. The density stratification is set by the momentum equation in conjunction with the mass-loss rate and the velocity field via the equation of continuity (Santolaya-Rey et al. 1997). For the models we used in this paper, the energy equation is decoupled from the radiative transfer problem by adopting a temperature structure based on the concept of nonLTE Hopf-like functions (see previous reference). In general, we do not allow a deviation larger than $4 \%$ of the flux conservation for models in the coarse grid (see below), while we ensure at least a flux conservation better than $2 \%$ for final models.

A description of the FASTWIND version used in this paper can be found in Santolaya-Rey et al. (1997) and Puls et al. (2003). The reader is referred to Puls et al. (2005) for a detailed discussion of the current status of the code and for a detailed comparison to similar codes. 
TABLE 2

Model Atoms Included in the Analysis

\begin{tabular}{|c|c|}
\hline Species & Reference \\
\hline 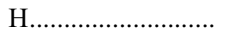 & Jokuthy (2002) \\
\hline $\mathrm{He}$ I/II ................... & Jokuthy (2002) \\
\hline 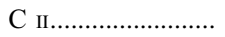 & Eber \& Butler (1988) \\
\hline 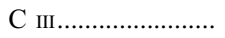 & Eber (1987) \\
\hline $\mathrm{N}$ II $\ldots \ldots \ldots \ldots \ldots \ldots \ldots \ldots$ & Becker \& Butler (1989) \\
\hline $\mathrm{N}$ III......................... & Butler (1984) \\
\hline 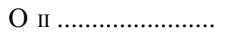 & Becker \& Butler (1988) \\
\hline 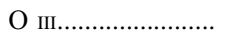 & D. Kunze (1998, private communication) \\
\hline $\mathrm{Mg}$ I/II .................. & K. Butler (1998, private communication) \\
\hline $\mathrm{Si} \mathrm{II/III/IV} \mathrm{..............}$ & Becker \& Butler (1990) \\
\hline
\end{tabular}

\subsubsection{Model Atoms}

Table 2 presents the chemical species considered in our models, along with the references where a complete description can be found. In general, the model atoms have been imported from the DETAIL code (Butler \& Giddings 1985), including in most cases several updates and revisions (Urbaneja 2004). The main difference with respect to the original versions is that FASTWIND includes currently a very approximate description of the dielectronic recombination process, known to be important for specific lines of C III and $\mathrm{N}_{\text {III }}$ (Mihalas et al. 1972) in B0 and earlier spectral types.

The particular configuration of each model atom has been chosen in order to describe properly the ionization balance of the species and to reproduce the optical line spectrum in the domain of early to mid B types. Therefore, model atoms for $\mathrm{C}, \mathrm{N}$, and $\mathrm{O}$ consider detailed data for II/III ionization stages plus the ground energy term of the IV ion; $\mathrm{Mg}$ is described by means of I/II ions plus the ground term of the III ion, while for Si we included II/III/IV plus the ground level of Si v.

\subsection{Model Grid}

Each FASTWIND model is specified by a set of parameters: the effective temperature, the surface gravity, the stellar radius (these three quantities are defined at Rosseland optical depth $\tau_{\mathrm{R}}=\frac{2}{3}$ ), the wind terminal velocity, the mass-loss rate, the $\beta$-exponent of the wind velocity law (for a definition see Santolaya-Rey et al. 1997), the helium abundance, the velocity of microturbulence, and a set of metal abundances. Therefore, even for a basic pure hydrogenhelium model atmosphere (without background elements), at least eight parameters are needed, which at a first glance could be considered a serious drawback for the analysis. However, not all these parameters have the same relevance in setting up the atmospheric structure. In order to reduce the space of parameters to be explored in the coarse grid, we fix some of them by introducing several considerations.

We assume a mean characteristic relationship between the spectral type (SpT) and the wind terminal velocity, following Haser (1995). The mean SpT- $v_{\infty}$ relationship is taken from Kudritzki $\&$ Puls (2000), which also requires the application of an underlying SpT- $T_{\text {eff }}$ scale (this $T_{\text {eff }}$ scale is only used as a guideline and is not important for the determination of the other stellar parameters). As has been shown by Puls et al. (1996, 2003), for a given wind terminal velocity, the mass-loss rate and the stellar radius can be combined in a unique parameter $Q^{\prime}=\dot{M} R_{\star}^{-1.5}$ that describes the wind strength. However, for a given fit of the $\mathrm{H} \alpha$ profile, an increase of $\beta$ can be compensated by a decrease of $Q^{\prime}$ (assuming that the $\mathrm{S} / \mathrm{N}$ is not very high). Thus, we need to fix $\beta$ to a suitable value for our objects. We take a representative value for each SpT bin from the results of the analyses of Galactic B-type supergiants by Kudritzki et al. (1999). The guess value is later improved during the fine-tuning analysis of each target (see $\S 3.3 .1)$.

Therefore, in this first step using a coarse grid the number of free parameters has been reduced from eight to five, plus the set of metal abundances. The next step is the description of the metal abundances, for which we assume a solar abundance pattern. It must be pointed out that the solar abundance pattern is not universal, as expected from the distribution of the ratio of the $\alpha$-elements to iron group elements (for a review see McWilliam 1997) and as recent works based on the analysis of red giants in nearby dSph galaxies (Shetrone et al. 2003; Tolstoy et al. 2003) have shown. Nevertheless, we construct the models assuming the solar abundances by Grevesse \& Sauval (1998), i.e., $8.52 \pm$ 0.06 dex for C, $7.92 \pm 0.06$ dex for $\mathrm{N}, 7.58 \pm 0.01$ dex for $\mathrm{Mg}$, and $7.56 \pm 0.01$ dex for $\mathrm{Si}$, except for the oxygen abundance, for which we choose the value from Allende Prieto et al. (2001), $8.69 \pm 0.05 \mathrm{dex}$. It is important to note that this particular choice has no effect on the atmospheric structure as long as the iron group elements (mainly Fe III to Fe v ions) are the dominant species for the background opacities. The abundances are scaled by the global metallicity for models with metallicities above/below the solar one. The assumption of a solar-like abundance pattern is retained during the fine-tuning abundance analysis for those elements for which we cannot get any information from the observed spectra, which presently means that only the abundances of helium, carbon, nitrogen, oxygen, magnesium, and silicon are updated to reproduce the observations.

Helium, being the second most abundant element, requires special care, as it influences the atmospheric structure (by means of a change in the molecular weight). Then, a change in the helium abundance could require some (few) iterations of the stellar parameters. As it has been discussed by McErlean et al. (1998), some helium lines in B stars are sensitive to the microturbulence velocity, meaning that, ideally, both helium abundance and atmospheric microturbulence velocity can be fixed at the same time. Unfortunately, only for earliest B types does helium exhibit lines from two ionization stages, He I/He II. Therefore, another chemical element is needed in order to determine the effective temperature (usually other elements are also preferred for determining $\left.v_{\text {turb }}\right)$. We adopt a solar helium abundance $[n(\mathrm{He}) / n(\mathrm{H})$ by number equal to 0.10$]$, fixing also $v_{\text {turb }}$ to $15 \mathrm{~km} \mathrm{~s}^{-1}$.

To summarize, four of the initial nine parameters have been fixed (He abundance, $v_{\text {turb }}, v_{\infty}$, and $\beta$ ) and we have adopted a relation for two others ( $\dot{M}$ and $R$, by using $Q^{\prime}$ ); thus, only four parameters (effective temperature, surface gravity, $Q^{\prime}$, and the global metallicity $Z$ for the background elements) remain to be covered by the coarse grid of models. Because the primary goal of this grid is the analysis of B0- to B5-type supergiant stars, we computed models in the range of 30,000-15,000 K, in steps of $1000 \mathrm{~K}$, with three different gravities (spaced in increments of 0.2 dex) for each $T_{\text {eff }}$ bin and three different values of $Q^{\prime}$ for each $T_{\text {eff }}-\log g$ pair. Six different sets of metal abundances were considered, corresponding to global metallicities of $1.4,1.0,0.7,0.5$, 0.3 , and $0.2 Z_{\odot}$.

\subsection{Methodology}

Equivalent widths (EWs) of metallic features were measured by nonlinear least-squares Gaussian fits to the observed lines. In case of blends, two or more Gaussian profiles were fitted simultaneously, allowing for free centering and width. The main source of uncertainties for the determination of the EW at the characteristic $\mathrm{S} / \mathrm{N}$ of our observational data set is the selection of 


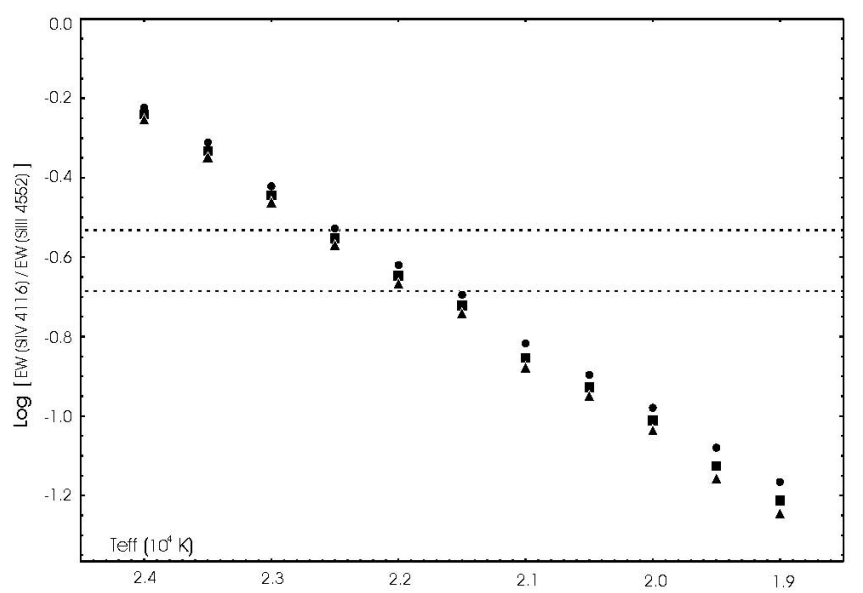

FIG. 5.-Effect of the microturbulence in the ratio of Si IV $\lambda 4116$ to Si III $\lambda 4552$. The measured ratio of a star in the sample is compared with the synthetic ratios at different turbulent velocities, obtained from the model grid (note that the $y$-axis is in $\log$ units). Ratios were evaluated at $v_{\text {turb }}=10$ (triangles), 15 (squares), and $20 \mathrm{~km} \mathrm{~s}^{-1}$ (circles). For each $T_{\text {eff }}$, we show only one of the surface gravities considered in the grid of models (representative of Ia luminosity class).

the continuum level. We decided to fit Gaussian profiles shifting the local continuum between $\pm 1 /(\mathrm{S} / \mathrm{N})$, taking as uncertainties the dispersion of the fitted values (following the ideas in Villamariz et al. 2002).

The silicon ionization balance is used as an indicator of the effective temperature of B-type stars. Ratios of observed EWs of lines of Si III to Si II (below B2 type) or Si IV to Si III (from B0 to B2 types) are compared to those obtained from the coarse model grid, giving the range of effective temperature in which the ratios are reproduced. The individual strengths of these lines are dependent on the microturbulent velocity, but the ratios are almost insensitive to $v_{\text {turb }}$ (see Fig. 5). As expected, larger differences are found when the strengths of lines from the two ionization stages are quite different because then the lines are on different parts of the curves of growth. Even in those cases the effect is small and leaves the temperature almost unaffected. On the other hand, these $\mathrm{EW}$ ratios can depend on stellar gravity; therefore, $T_{\text {eff }}$ and surface gravity need to be obtained simultaneously by means of an iterative process.

The surface gravity for each temperature is given by fits to the hydrogen Balmer lines. These lines are dominated by Stark broadening, and they are almost unaffected by microturbulent line broadening (McErlean et al. 1999). However, the procedure to obtain the surface gravity is a bit more elaborated than in the case of plane-parallel, hydrostatic model atmospheres, since our models also consider the effects of the stellar winds, which can strongly affect the Balmer lines and, therefore, the determination of gravities. There are two effects that can modify the profiles due to the mass outflow (Puls et al. 1996): First, at optical depth unity, the mass density is lower in a unified model than in a plane-parallel model, and consequently the Stark broadening becomes less effective, leading to narrower lines. Second, the wind emission can refill the photospheric profile. In cases of weak winds, surface gravities obtained by means of planeparallel, hydrostatic models do not differ from the ones determined from unified models. Basically, the density stratifications are identical and there is a negligible wind emission. As the wind increases and larger values of $\dot{M} / v_{\infty} R_{\star}^{2}$ are reached, the sonic point moves inward, toward (or even deep inside) the photosphere; the density stratification is modified, being always lower in the unified model (Gabler et al. 1989), and the wind emission in- creases significantly. As a consequence, the hydrogen Balmer lines are affected. Moreover, the determinations of effective temperatures are also affected, since a shift in gravity changes the intersection in $T_{\text {eff }}-\log g$ plane with the fit curve for the silicon ionization equilibrium. Usually we encounter good agreement between plane-parallel and unified model fit results in the case of weak winds; however, there are increasing differences as the strength of the wind and the importance of the atmospheric extension grow. Thus, while models for dwarf stars lead to nearly identical results, this is not generally true for the case for supergiant stars for which the results will depend on each particular case.

The mass-loss rate and the exponent of the wind velocity law, $\beta$, follow from the fits of the $\mathrm{H} \alpha$ profiles (see $\S$ 3.3.1). As previously discussed, the mass-loss rate is linked to the stellar radius via $Q^{\prime}$. The stellar radius is obtained in the usual way from the absolute magnitude of the star deduced from the apparent magnitude after the adoption of the distance modulus and the correction for interstellar extinction, which is obtained from the reddening (assuming a standard value of $R_{v}=3.1$ for the relationship between the color excess and the visual extinction) and from the emergent flux of the model (Kudritzki 1980). While observing in other galaxies has the advantage of a lower relative distance uncertainty as compared to the Milky Way, as well as a lower extinction in many cases, it also poses additional problems concerning the photometry. The most important one is the crowding, which may contaminate not only the stellar spectrum but also, more importantly, the measured apparent magnitude. Accurate photometry is therefore crucial for extragalactic stellar work. Unfortunately, there is not yet a uniform photometry of the whole galaxy, and we have to rely on different sources for these quantities. Figure 6 presents a comparison of the photometric magnitudes for the stars in our sample included in the photometric surveys of Ivanov et al. (1993) and Massey et al. (1996). In general, the agreement on $m_{V}$ is good, while a systematic effect seems to be present in the comparison of the $B$-band magnitudes. However, the difference between $B-V$ is small and not important for the stars in our sample (the color excess and hence the visual extinction would be somewhat lower for 110A and 0900 with the Massey et al. [1996] results, but the differences will not introduce any significant effect on derived related stellar quantities).

With the initial set of parameters known (effective temperature, surface gravity, stellar radius, mass-loss rate, wind terminal velocity, $\beta$ ), along with the helium abundance fixed at solar value, new models are calculated (an individual fine grid), varying the silicon abundance and the microturbulence. Both quantities are fixed simultaneously at the value for which no dependence in the derived abundance with the strength of the lines is found. At the same time, this procedure gives us the uncertainties in microturbulence and abundance. Finally, the initial assumption about the helium abundance is checked by comparing synthetic and observed $\mathrm{He}$ I/He II lines. The analysis process is started from the beginning if the helium abundance has to be updated.

The abundance analysis methodology outlined for Si, based on the curve of growth (Gray 1982), is used for those elements for which a number of spectral lines are observed ( $\mathrm{N}$ and $\mathrm{O}$ ), allowing the determination of an independent microturbulence, which can be compared with the one obtained from the silicon lines. In the case that a zero-slope fit cannot be found for an element, the abundance is evaluated at the microturbulence given by the Si lines. Note that for carbon and magnesium there is only one line present in the observed wavelength range, namely, 

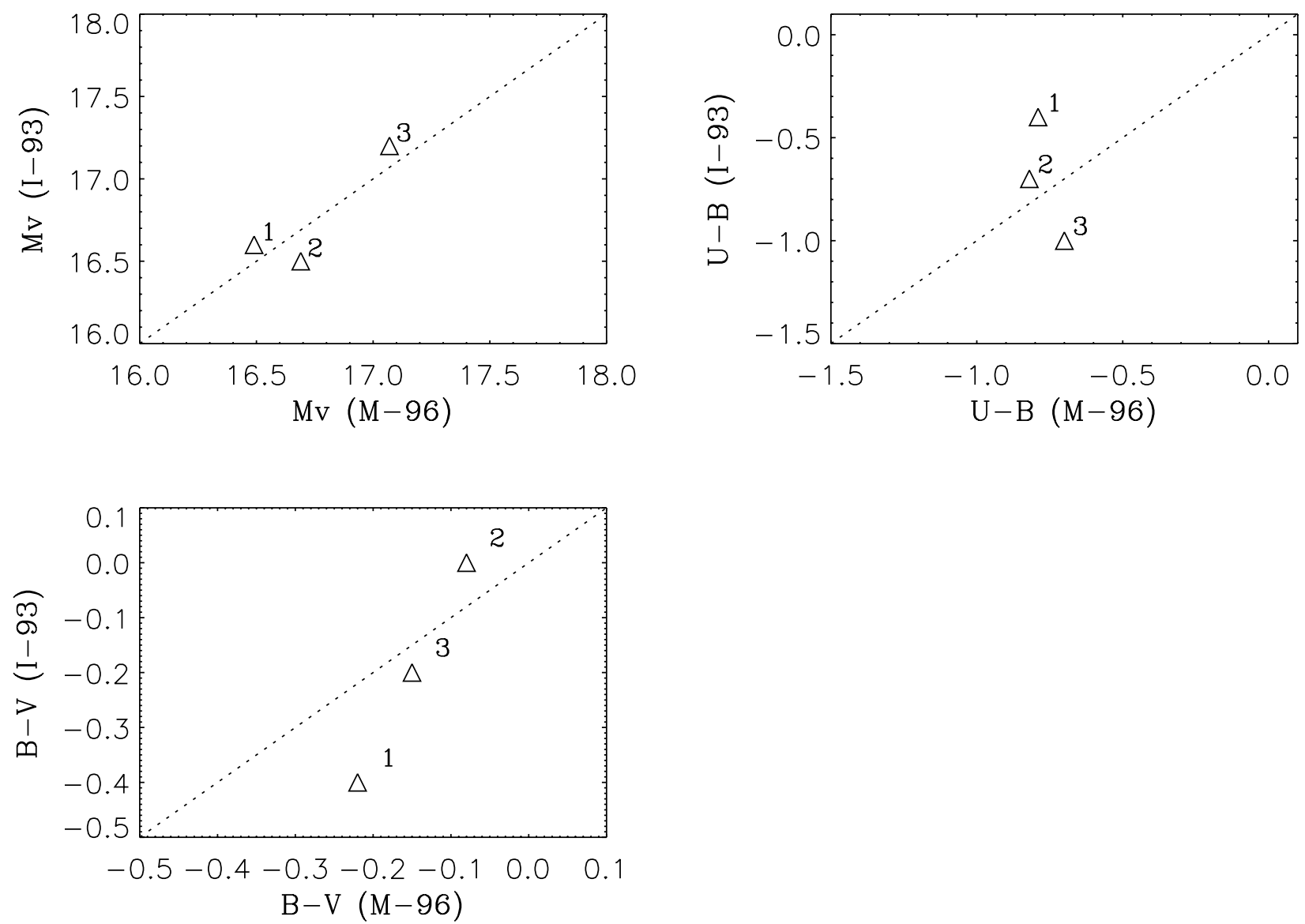

FIG. 6.-Comparison of photometric measurements from Ivanov et al. (1993) and Massey et al. (1996) for the stars in our sample. The stars are 1054 (1), B133 (2), and 1137 (3).

$\mathrm{C}_{\text {II }} \lambda 4267$ and $\mathrm{Mg}$ II $\lambda 4481$, which excludes those elements from a microturbulence analysis. As a general rule, we finally adopt the $v_{\text {turb }}$ given by the $\mathrm{Si}$ lines for all the elements.

\subsubsection{Mass-Loss Rates: Analysis of the $\mathrm{H} \alpha$ Profiles}

Several observational techniques can be used in order to determine the rate at which a blue massive star is losing mass, for example, the measurement of the IR or radio continuum excess. However, the analysis of the $\mathrm{H} \alpha$ profiles remains the fundamental technique for the derivation of $\dot{M}$ for extragalactic targets (see Kudritzki \& Puls 2000). $\mathrm{H} \alpha$ is a recombination line that is formed in an extended region of the atmosphere, which means that the line has a (significant) contribution from the stellar wind. By reproducing the profile we will, in principle, get information about $\beta$ (which constrains the wind density stratification via the velocity field and the equation of continuity) and $v_{\text {turb }}^{\text {wind }}$, the turbulent velocity in the stellar wind, which might be significantly different from the photospheric microturbulence. However, if the winds are weak and the line is in absorption, $\beta$ can only be constrained to a minimum value fixed by the rise of extended emission wings. Also, in emission cases, $\beta$ can be very well constrained from the width of the profile at half-maximum of the emission peak; the shape of the emission peak is mainly determined by the velocity field, showing low and broad emission for low values of $\beta$ and high and narrow emissions for high $\beta$ values. It is important to properly constrain $\beta$ since, for a given emission, a lower value of $\beta$ implies a larger $\dot{M}$. In case of emission, the location of the maximum can give some information about $v_{\text {turb }}^{\text {wind }}$.
There are several unsolved problems in modeling the $\mathrm{H} \alpha$ profiles of particular stars and several observational limitations that can affect the interpretation of the profiles. First, we have already quoted the poor constraint of $\beta$ when the line is in absorption (although in these cases the influence of the wind is also smaller). For some stars, it is not possible to reproduce the weak blue absorption sometimes present, although the emission is well fitted. This may indicate the presence of clumped material in the wind or an imperfect description of the velocity field. For very high rates of mass loss it is not possible to reproduce simultaneously all of the Balmer lines; in addition, these stars exhibit mass-loss rates much larger than predicted by the theory of radiation-driven winds, which is interpreted again as the presence of inhomogeneities in the wind (Herrero et al. 2002; Repolust et al. 2004). An observational problem in the analysis of $\mathrm{H} \alpha$, independent of the models, is the contamination of the stellar profile by the emission of the surrounding ISM. This problem is particularly important for extragalactic stars, usually observed at lower $\mathrm{S} / \mathrm{N}$ and spectral and spatial resolutions (with a larger emitting volume in the slit) if optically thin ISM gaseous matter is present.

\subsubsection{Diagnostic Lines}

The 3970-4750 $\AA$ wavelength range of B0- to B5-type supergiant spectra, apart from $\mathrm{H}$ and $\mathrm{He}$ lines, is dominated by the presence of $\mathrm{O}$ II lines. However, a considerable number of $\mathrm{N}$ II / N III, Si II/Si III/Si IV, C II/C III, and Mg II lines are also present, providing valuable information about chemical composition. A few weak lines from other species (like, for example, Fe III 
and $\mathrm{Al}$ III) can be observed, but only with very high $\mathrm{S} / \mathrm{N}$, which we were not able to obtain for our sample.

The $\mathrm{H}$ Balmer lines $\mathrm{H} \gamma, \mathrm{H} \delta$, and $\mathrm{H} \epsilon$ are observed in the blue range, plus $\mathrm{H} \alpha$ in the red spectral range. $\mathrm{H} \epsilon$ is discarded because of the blend with the ISM (and possibly a stellar component) $\mathrm{Ca}$ II $(\mathrm{K})$ line. The blue wing of $\mathrm{H} \delta$ is affected by the presence of the $\mathrm{N}$ III $\lambda 4097$ line, while blue and red wings of $\mathrm{H} \gamma$ contain several $\mathrm{O}$ II lines (as well as an $\mathrm{N}$ III line in the blue wing). The presence of such blends, if not resolved or not accounted for, can affect the determination of the surface gravity.

A large number of helium lines are available in the observed wavelength window. However, not all of these lines are used to determine the $\mathrm{He}$ abundance, for different reasons. Primary lines we consider in the analysis are the He I $\lambda \lambda 4026,4713$ lines (both belong to the triplet spin system) and $\lambda 4387$ (which is a singlet line). For those stars with a large spectral coverage, also $\lambda 4922$ (singlet spin system) is used, while $\lambda \lambda 6678$ and 4471 (singlet and triplet systems, respectively), although observed, are not directly considered to provide constraints for the analysis (the reader is referred to $\S 3.5 .3$ for a detailed discussion of the problems found in modeling individual lines). The lines $\lambda \lambda 4009$ (singlet), 4120 (triplet), 4143, and 4437 (both singlet lines) are also not taken into account to constrain the analysis because the Stark broadening data available to us do not seem accurate enough. However, they are included in the final fits shown later. For B0 stars three He II lines are generally observed; of those $\lambda \lambda 4200$ and 4541 are used for the analysis, while $\lambda 4686$ is not (see $\S 3.5 .3$ ).

Carbon abundances are based on only one line, the problematic C II $\lambda 4267$ line (see $\S 3.5 .3$ ), since the $\mathrm{C}_{\text {II }} \lambda \lambda 6578,6582$ lines are only observed for two stars (OB 10-10 and 1054).

Lines from single ionized $\mathrm{N}$ are used to determine $\mathrm{N}$ abundances. Mainly, these lines are $\lambda \lambda 3995,4237,4241,4447,4601$, $4607,4614,4621$, and 4630 . Several N III lines ( $\lambda \lambda 4097,4379$, and 4634) are strong enough to be detected in the earlier spectra (at our $\mathrm{S} / \mathrm{N}$ ), but we do not use these lines as diagnostic tools because of the approximate treatment of dielectronic recombinations in our models (Urbaneja 2004).

Single ionized oxygen lines dominate the optical spectra of early to mid B-type supergiants, including strong and weak lines, which means that $\mathrm{O}$ can be used to determine the microturbulence. In practice, microturbulence velocities derived from $\mathrm{O}_{\text {II }}$ lines are systematically larger (of the order of $\sim 5 \mathrm{~km} \mathrm{~s}^{-1}$ ) than the ones obtained from the Si III lines. Most likely, this difference does not reflect any physical effect and is probably related to the uncertainties of the atomic quantities involved (uncertainties in oscillator strengths are generally above the $10 \%-20 \%$ level). The lines considered in our analyses are $\lambda \lambda 4069,4072$, $4078,4317,4319,4366,4591,4596,4414,4416$, and 4696, which seem to be unblended in the parameter range of this work.

Magnesium abundances also are based on only one line, $\mathrm{Mg}$ II $\lambda 4481$. For both $\mathrm{C}$ and $\mathrm{Mg}$ abundances, the uncertainties given in the tables below represent uncertainties only due to the $\mathrm{S} / \mathrm{N}$. The possible contribution of the $\mathrm{Al}$ III $\lambda 4479$ line to the spectral feature observed at $4481 \AA$ cannot be resolved at the spectral resolution of our spectra, in particular at $R=2500$. Recently, Lyubimkov et al. (2005) have shown that the ratio of observed EWs of $\mathrm{Al}$ III to $\mathrm{Mg}$ II lines can reach the level of $30 \%$ in Galactic B-type dwarf stars. However, from an exploratory study of our Galactic high-resolution spectra, we could conclude that the contribution, if any, is only marginal. Besides, the presence of a relatively important $\mathrm{Al}$ III $\lambda 4479$ line would be accompanied by a spectral feature (not observed unless perhaps for B133) located at $4530 \AA$, formed by the $\mathrm{Al}$ III $\lambda \lambda 4528,4259$ lines and $\mathrm{N}$ II
24530. Therefore, the derived Mg abundances will be presumably not affected by the blend with $\mathrm{Al}$ III, except perhaps in the case of B133.

Finally, lines for at least two consecutive ionization stages of silicon are generally present in the observed spectra, with a number of cases in which lines from the three Si ions can be detected. The diagnostic lines, used for $T_{\text {eff }}$, microturbulence, and $\mathrm{Si}$ abundances, are Si II $\lambda \lambda 4128,4130, \mathrm{Si}$ III $\lambda \lambda 4552,4567$, 4574, and Si Iv $\lambda \lambda 4089,4416$.

The wider spectral wavelength coverage of the Keck ESI observations provides us with additional diagnostic lines; for B0-like stars, in the 5000-6000 $\AA$ wavelength range, it is possible to use three $\mathrm{He} \mathrm{I}$ lines $(\lambda \lambda 5010,5050$, and 5875$)$ plus one $\mathrm{He}$ II line at $5410 \AA$; along with these, other metal lines are available: an Si III line at $5746 \AA$ and a particularly interesting O III line (the only one observed) at $5592 \AA$, which will allow for the use of the $\mathrm{O}$ II $/ \mathrm{O}$ III ionization balance for constraining $T_{\text {eff }}$. For cooler types, as is the case for $\mathrm{OB} 10-10$, strong $\mathrm{N}$ II lines are observed at 5000-5010 and $5045 \AA$, blended in some cases with the He I lines; also in the range 5650-5750 Å we can find strong $\mathrm{N}$ II lines, together with $\mathrm{Al}$ III and, possibly, C III lines. Finally, a few $\mathrm{Fe}$ III lines appear in the spectrum, which will make the determination of $\mathrm{Fe}$ abundances possible in future work. Included also in the ESI coverage is $\mathrm{H} \beta$, which allows a further constraint for $\dot{M}$, as well as $\mathrm{He} \mathrm{I} \lambda 4922$.

\subsection{Uncertainties}

Several quantities derived depend primarily on the quality of the observed spectra, like the effective temperatures or the surface gravities. Others, like radii or luminosities, depend also on photometric data, while the mass-loss rates and the chemical abundances are dependent on the values found for the other fundamental parameters.

At an $\mathrm{S} / \mathrm{N}$ of $\sim 60, T_{\text {eff }}$ can be typically constrained to $\pm 1000 \mathrm{~K}$ for spectra obtained with the lowest spectral resolution, while the uncertainty is reduced to $\pm 500 \mathrm{~K}$ for the stars observed with the highest spectral resolution. Generally speaking, this also depends on the spectral type, the uncertainty being larger for those stars located in the regions of the $T_{\text {eff }}-\log g$ plane where the transition of the silicon dominant species, from $\mathrm{Si}$ IV/III to $\mathrm{Si}$ III/II, occurs (i.e., when the lines from both ions $\mathrm{Si}$ IV and $\mathrm{Si}$ II are weak simultaneously, around B1.5 spectral type). Surface gravities can be very well determined from the simultaneous fits of different $\mathrm{H}$ Balmer lines, generally to an uncertainty of \pm 0.05 dex. However, we also have to consider the contribution of the uncertainty in the mass-loss rate (affected by the uncertainties in $\beta, v_{\infty}$, and the final derived stellar radius). Two other considerations have to be included. First, we are assuming that the line list we use for the blanketing (see Puls et al. 2005) is complete, i.e., that the photospheric line pressure is properly accounted for. And second, we are assuming that the Fe abundances scale with the global metallicity in a linear way. There is not an easy way to estimate the completeness of the blanketing line list; however, from comparisons with other model atmosphere codes (the reader is referred to the later reference) it seems to be fairly complete in the domain covered in our analysis. Concerning the scaling of $\mathrm{Fe}$ abundances, models computed varying $\mathrm{Fe}$ by -0.2 dex do not show any significant effect on the Balmer $\mathrm{H}$ line profiles. Thus, we conclude that the typical uncertainty for surface gravities reaches the level of $0.10-0.15$ dex, depending on the wind strength.

The accuracy of the stellar radius depends strongly on accuracy of the photometric quantities, like the apparent visual 
TABLE 3

Abundance Uncertainties Obtained from the Grid of Models: Model 24,000/2.60

\begin{tabular}{|c|c|c|c|c|c|c|c|}
\hline Element & Line & $\begin{array}{c}\Delta \log g \\
( \pm 0.15 \text { dex })\end{array}$ & $\begin{array}{c}\Delta T_{\mathrm{eff}} \\
( \pm 1000 \mathrm{~K})\end{array}$ & $\begin{array}{c}\Delta v_{\text {turb }} \\
\left(-5 \mathrm{~km} \mathrm{~s}^{-1}\right)\end{array}$ & $\begin{array}{c}\Delta \mathrm{He} \\
(+0.2 \mathrm{dex})\end{array}$ & $\begin{array}{c}\Delta Z \\
(-0.2 \operatorname{dex})\end{array}$ & $\begin{array}{c}\Delta \log Q \\
( \pm 0.2 \mathrm{dex})\end{array}$ \\
\hline \multicolumn{8}{|c|}{$\sigma(\mathrm{C})=0.23 \mathrm{dex}$} \\
\hline 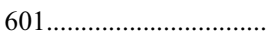 & $\lambda 4267$ & 0.11 & 0.18 & 0.10 & 0.02 & 0.01 & 0.01 \\
\hline \multicolumn{8}{|c|}{$\sigma(\mathrm{N})=0.21 \mathrm{dex}$} \\
\hline 701......................... & $\lambda 3995$ & 0.08 & 0.18 & 0.07 & 0.02 & 0.02 & 0.03 \\
\hline 701......................... & $\lambda 5025$ & 0.03 & 0.17 & 0.06 & 0.05 & 0.06 & 0.06 \\
\hline 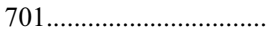 & $\lambda 5045$ & 0.02 & 0.16 & 0.06 & 0.05 & 0.04 & 0.05 \\
\hline \multicolumn{8}{|c|}{$\sigma(\mathrm{O})=0.14 \mathrm{dex}$} \\
\hline 801 & $\lambda 4069$ & 0.02 & 0.07 & 0.06 & 0.01 & 0.00 & 0.00 \\
\hline 801........................ & $\lambda 4072$ & 0.01 & 0.07 & 0.11 & 0.01 & 0.00 & 0.00 \\
\hline 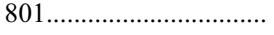 & $\lambda 4076$ & 0.01 & 0.06 & 0.13 & 0.01 & 0.01 & 0.00 \\
\hline 801........................ & $\lambda 4317$ & 0.03 & 0.07 & 0.07 & 0.04 & 0.02 & 0.03 \\
\hline 801 & $\lambda 4319$ & 0.03 & 0.06 & 0.07 & 0.03 & 0.01 & 0.02 \\
\hline 801 & $\lambda 4414$ & 0.04 & 0.04 & 0.10 & 0.02 & 0.02 & 0.00 \\
\hline $801 \ldots \ldots$ & $\lambda 4416$ & 0.03 & 0.06 & 0.08 & 0.03 & 0.01 & 0.01 \\
\hline \multicolumn{8}{|c|}{$\sigma(\mathrm{Mg})=0.21 \mathrm{dex}$} \\
\hline $1201 \ldots$ & $\lambda 4481$ & 0.09 & 0.18 & 0.07 & 0.01 & 0.02 & 0.02 \\
\hline
\end{tabular}

NotEs.-The influence of the uncertainties in each fundamental parameter is considered. The global abundance uncertainty for each element is obtained as the square root of the sum of the squares of these uncertainties, assuming them to be independent. The element identification is codified as 100 times atomic number plus the ionization stage; for example: 701 corresponds to singly ionized nitrogen.

magnitude, colors, and the adopted ratio between reddening and visual extinction, and, of course, of the adopted distance. The stellar radius is also important to determine the stellar luminosity and the spectroscopic stellar mass (the latter is derived from the gravity), which are fundamental in the comparison with evolutionary models. In the case of Galactic stars, the knowledge of distance is the major source of uncertainty, whereas for objects in other galaxies, at least the relative distances are well known. However, crowding and multiplicity become a problem, in particular in cases where high spatial resolution images are not available. As we have seen above, several of the observed M33 stars have close companions that have the potential to affect the stellar radius derived (and the related quantities).

The determination of mass-loss rate and wind momentum depends strongly on the quality and nature of the $\mathrm{H} \alpha$ profile. The $\mathrm{S} / \mathrm{N}$, whether the profile is in emission or absorption, and the presence, strength, and relative location of $\mathrm{H}$ II components are all crucial factors. In addition, the mass-loss rate derived depends on $v_{\infty}$ and (slightly) on $v_{\text {turb }}$.

The main source of individual uncertainties for chemical element abundances is the $\mathrm{S} / \mathrm{N}$ achieved during the observations. In addition, the continuum normalization and, of course, the uncertainties of the stellar parameters may be of importance. In order to determine the metal abundance uncertainties, we take into account the uncertainties in the effective temperature, the surface gravity (which included the effect of wind parameters), the microturbulence, and the uncertainty in the helium abundance, if any, as well as the internal dispersion between the different line abundances. In the following tables, the abundance uncertainties are split into two contributions, the line-to-line scatter and an estimation of the systematic uncertainties obtained from the model grid (see Tables 3 and 4 for two limiting cases). We also explore the effect of uncertainties in atomic quantities in Table 5, showing that these produce a systematic uncertainty at the level of $\sim 0.1$ dex.

\subsection{Individual Comments}

\subsubsection{Stars in the WHT ISIS Data Set}

The star 0900 , or B217a, is affected by strong H II region emission almost centered at the rest wavelength of the stellar $\mathrm{H}$ Balmer lines: the two observed $\mathrm{H}$ lines, $\mathrm{H} \gamma$ and $\mathrm{H} \delta$, are heavily distorted. Therefore, the results obtained have to be regarded more as an estimate rather than as accurate values, in particular for the wind parameters. For the determination of metallicity, because of the low quality of the spectrum, we apply a combination of two analysis techniques, using simultaneously the equivalent widths of the strongest lines and the comparison of the whole spectrum with synthetic ones, looking for the best match (Urbaneja et al. 2003, 2005). We find a metallicity close to solar. The stellar parameters derived are consistent with our initial classification as a B0.5-B1 Ia star.

Object 110A, the star 0785 of Ivanov et al. (1993), shows strong emission profiles in $\mathrm{H} \alpha$ and $\mathrm{H} \beta$, with $\mathrm{H} \gamma$ as a P Cygni profile and $\mathrm{H} \delta$ partially filled by stellar wind emission. Higher Balmer lines are also affected. Note that $\mathrm{H} \epsilon$ shows an enhanced absorption, which is due to the IS Ca II $(\mathrm{K})$ line, consistent with the strong absorption at the location of the $\mathrm{Ca}$ II $(\mathrm{H})$ line at $3933.67 \AA$. This is also in agreement with the results obtained for the $\mathrm{H}$ I mass column densities (see Table 6), with 110A showing one of the highest values. The star appears like an isolated object in HST Space Telescope Imaging Spectrograph (STIS) acquisition images (Fig. 7; GO program 7481, PI: Kudritzki). Object $110 \mathrm{~A}$ represents an extreme case of stars with high-density winds. The mass-loss rate deduced from the fit of $\mathrm{H} \alpha$ and $\mathrm{H} \beta$ is very high, and all other Balmer lines are also affected by wind emission. 
TABLE 4

Abundance Uncertainties Obtained from the Grid of Models: Model 21,000/2.50

\begin{tabular}{|c|c|c|c|c|c|c|c|}
\hline Element & Line & $\begin{array}{c}\Delta \log g \\
( \pm 0.15 \text { dex })\end{array}$ & $\begin{array}{c}\Delta T_{\text {eff }} \\
( \pm 1000 \mathrm{~K})\end{array}$ & $\begin{array}{c}\Delta v_{\text {turb }} \\
\left(-5 \mathrm{~km} \mathrm{~s}^{-1}\right)\end{array}$ & $\begin{array}{c}\Delta \mathrm{He} \\
(+0.2 \mathrm{dex})\end{array}$ & $\begin{array}{c}\Delta Z \\
(-0.2 \mathrm{dex})\end{array}$ & $\begin{array}{c}\Delta \log Q \\
( \pm 0.2 \text { dex }\end{array}$ \\
\hline \multicolumn{8}{|c|}{$\sigma(\mathrm{C})=0.22 \mathrm{dex}$} \\
\hline $601 \ldots \ldots \ldots \ldots \ldots \ldots$ & $\lambda 4267$ & 0.09 & 0.13 & 0.15 & 0.01 & 0.00 & 0.02 \\
\hline \multicolumn{8}{|c|}{$\sigma(\mathrm{N})=0.20 \mathrm{dex}$} \\
\hline $701 \ldots \ldots \ldots \ldots \ldots \ldots$ & $\lambda 3995$ & 0.10 & 0.14 & 0.10 & 0.02 & 0.01 & 0.02 \\
\hline 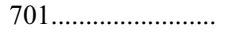 & $\lambda 5025$ & 0.02 & 0.16 & 0.06 & 0.07 & 0.06 & 0.06 \\
\hline $701 \ldots \ldots \ldots \ldots \ldots \ldots \ldots$ & $\lambda 5045$ & 0.00 & 0.15 & 0.07 & 0.05 & 0.03 & 0.04 \\
\hline \multicolumn{8}{|c|}{$\sigma(\mathrm{O})=0.25 \mathrm{dex}$} \\
\hline $801 \ldots \ldots \ldots \ldots \ldots \ldots . .$. & $\lambda 4069$ & 0.20 & 0.07 & 0.08 & 0.03 & 0.01 & 0.01 \\
\hline 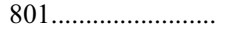 & $\lambda 4072$ & 0.21 & 0.08 & 0.09 & 0.03 & 0.01 & 0.01 \\
\hline 801................................. & $\lambda 4076$ & 0.20 & 0.09 & 0.09 & 0.03 & 0.01 & 0.01 \\
\hline $801 \ldots \ldots \ldots \ldots \ldots \ldots \ldots$ & $\lambda 4317$ & 0.20 & 0.05 & 0.09 & 0.06 & 0.03 & 0.02 \\
\hline 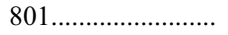 & $\lambda 4319$ & 0.20 & 0.05 & 0.09 & 0.05 & 0.02 & 0.01 \\
\hline $801 \ldots \ldots \ldots \ldots \ldots \ldots \ldots . .$. & $\lambda 4414$ & 0.20 & 0.10 & 0.09 & 0.04 & 0.01 & 0.00 \\
\hline $801 \ldots \ldots \ldots \ldots \ldots \ldots \ldots \ldots$ & $\lambda 4416$ & 0.21 & 0.10 & 0.09 & 0.05 & 0.01 & 0.01 \\
\hline \multicolumn{8}{|c|}{$\sigma(\mathrm{Mg})=0.19 \mathrm{dex}$} \\
\hline $1201 \ldots \ldots \ldots \ldots \ldots \ldots \ldots$ & $\lambda 4481$ & 0.09 & 0.14 & 0.08 & 0.01 & 0.01 & 0.02 \\
\hline
\end{tabular}

Unfortunately, $\mathrm{H} \beta$ would probably require an even higher $\dot{M}$ than the one adopted, while $\mathrm{H} \gamma$ would suggest a lower massloss rate. This indicates a density stratification in the wind different from the one adopted with a simple $\beta$-law. It might also hint at the presence of clumped material in the wind. Derived parameters place the star well above the Humphreys-Davidson limit (Humphreys \& Davidson 1979), in a region of the HRD that is populated by LBV stars. However, 110A does not show the typical emission-line spectrum of such objects and appears more like an "extreme" supergiant.

B38, the star 108 in the association OB 21 (aka UIT 030), shows a nice blue absorption spectrum, although the red spectrum is affected by the emission from an $\mathrm{H}$ II region. The analysis does not present any major problem. We derive $\alpha$-element abundances representative of a mean metallicity of $0.7 Z_{\odot}$.

The HST STIS acquisition images (Fig. 7) resolve two objects in the position of B133; however, one of them is significantly fainter. The ground-based blue spectrum does not show any evidence of a composite object and indicates a normal B1B1.5 Ia star. The analysis of the optical spectrum does not pres- ent any particular problem, except for the emission in $\mathrm{H} \alpha$, which is not consistent with the other $\mathrm{H}$ Balmer lines. The surface gravity is well constrained from fits of the observed high Balmer lines. While all other metals seem to be normal, the fit of the magnesium line requires an unusually high abundance to reproduce the observed EW. The presence of a companion would affect the observed visual magnitude and consequently all of the physical quantities derived from its value: the stellar radius, the luminosity, and the spectroscopic mass. As discussed in Urbaneja et al. (2002), the UV spectrum shows several peculiarities, which could be interpreted as the presence of a second star in the slit. Unfortunately, the central wavelength and the large bandwidth of the STIS acquisition image do not allow us to constrain the spectral type of the fainter object.

Object 1054, or UIT 215, is an important object for the present study because it is the star closest to the center of M33. Images from the HST WFPC2 (GO program 6651, PI: McCarthy) partially resolve a small compact stellar group at the location of the star. However, as discussed by Monteverde et al. (2000), there is no strong evidence of contamination either in the blue or in the

TABLE 5

Estimation of the Effect of Atomic Quantity Uncertainties on Abundance Uncertainties

\begin{tabular}{|c|c|c|c|c|c|c|c|c|c|c|}
\hline \multirow[b]{2}{*}{ SOURCE } & \multicolumn{5}{|c|}{ Model $26,000 / 2.80$} & \multicolumn{5}{|c|}{ Model $21,000 / 2.50$} \\
\hline & $\mathrm{C}$ & $\mathrm{N}$ & $\mathrm{O}$ & $\mathrm{Mg}$ & $\mathrm{Si}$ & $\mathrm{C}$ & $\mathrm{N}$ & $\mathrm{O}$ & $\mathrm{Mg}$ & $\mathrm{Si}$ \\
\hline $1.1 \sigma_{\mathrm{rbf}} \ldots \ldots \ldots \ldots \ldots \ldots$ & -0.01 & -0.07 & -0.03 & -0.05 & -0.04 & -0.01 & -0.07 & -0.02 & -0.04 & -0.02 \\
\hline 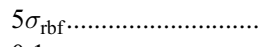 & -0.38 & -0.32 & -0.43 & -0.41 & -0.31 & -0.41 & -0.33 & -0.45 & -0.40 & -0.28 \\
\hline $0.1 \sigma_{\mathrm{cbf}} \ldots \ldots \ldots \ldots \ldots \ldots \ldots$ & 0.00 & -0.06 & -0.02 & -0.03 & -0.04 & 0.01 & -0.05 & 0.00 & -0.03 & -0.02 \\
\hline $10 \sigma_{\mathrm{cbf}} \ldots \ldots \ldots \ldots \ldots \ldots \ldots \ldots$ & 0.01 & -0.06 & -0.01 & -0.03 & -0.04 & 0.01 & -0.05 & 0.00 & -0.03 & -0.02 \\
\hline $0.5 \sigma_{\mathrm{cbb}} \ldots \ldots \ldots \ldots \ldots \ldots \ldots \ldots$ & 0.01 & -0.06 & -0.01 & -0.03 & -0.04 & 0.01 & -0.05 & 0.00 & -0.03 & -0.02 \\
\hline $2 \sigma_{\mathrm{cbb}} \ldots \ldots \ldots \ldots \ldots \ldots \ldots \ldots$ & 0.00 & -0.06 & -0.02 & -0.03 & -0.04 & 0.01 & -0.05 & 0.00 & -0.03 & -0.02 \\
\hline $1.1 f_{\mathrm{ij}} \ldots \ldots \ldots \ldots \ldots \ldots \ldots \ldots \ldots$ & 0.06 & -0.01 & 0.05 & 0.02 & 0.01 & 0.06 & 0.00 & 0.07 & 0.02 & 0.03 \\
\hline 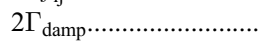 & 0.01 & -0.06 & -0.01 & -0.03 & -0.03 & 0.02 & -0.05 & 0.00 & -0.03 & -0.02 \\
\hline
\end{tabular}

Notes.-Typical uncertainties in radiative and collisional cross sections reach the level of $10 \%-20 \%$; we also explore larger variations for some atomic processes. Note that a large variation of the damping constants, poorly known actually, has almost no impact on the abundances, except for $\mathrm{N}$. 
TABLE 6

Gas-to-Dust Ratio for the Sample of M33 B-Type Supergiants

\begin{tabular}{|c|c|c|c|}
\hline Star & $\begin{array}{l}\log N\left(\mathrm{H} \mathrm{I}_{\mathrm{I}}\right) \\
\quad(\mathrm{dex})\end{array}$ & $\begin{array}{c}E(B-V) \\
\quad(\mathrm{mag})\end{array}$ & $\begin{array}{c}N(\mathrm{H} \mathrm{I}) / E(B-V) \\
\left(10^{22} \mathrm{~cm}^{-2} \mathrm{mag}^{-1}\right)\end{array}$ \\
\hline $0900 \ldots$ & $20.85 \pm 0.05$ & 0.07 & $1.01 \pm 0.43$ \\
\hline $110 \mathrm{~A} \ldots \ldots \ldots \ldots$ & $21.15 \pm 0.03$ & 0.19 & $0.74 \pm 0.11$ \\
\hline 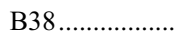 & $20.70 \pm 0.03$ & 0.10 & $0.50 \pm 0.15$ \\
\hline 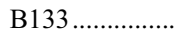 & $20.97 \pm 0.03$ & 0.09 & $1.04 \pm 0.35$ \\
\hline $1137 \ldots \ldots \ldots \ldots \ldots$ & $20.85 \pm 0.05$ & 0.01 & $7.08 \pm 0.82$ \\
\hline UIT $122 \ldots \ldots \ldots . .$. & $21.10 \pm 0.10$ & 0.09 & $1.40 \pm 0.32$ \\
\hline
\end{tabular}

Note.-Neutral H column densities taken from the UV analysis by Urbaneja et al. (2002) and Urbaneja (2004) are combined with the color excess obtained in our analysis of the optical spectra.

red spectra, both looking quite normal. The only doubt arises from the absorption features located in the wavelength range 4125-4140 $\AA$. If these are assumed to be the $\mathrm{Si}$ II doublet, then they appear blueshifted by more than $90 \mathrm{~km} \mathrm{~s}^{-1}$ with respect to other Si lines present in the spectra. On the other hand, and bearing in mind that the star is close to the galactic center, for which a high metallicity could be expected (and in particular a high oxygen abundance), both features can be interpreted as $\mathrm{O}$ II lines located at 4129.3 and $4132.8 \AA$ (multiplet number 107 in NIST database); actually, at least the strong absorption at $4132 \AA$ is reproduced by our final model (for the oxygen abundance derived from other $\mathrm{O}$ II strong lines). As for the case of B133, the visual magnitude will be affected by the presence of the companions, being also compromised by the derived radius, lumi- nosity, and stellar mass. On the other hand, a strong influence on the effective temperature, the surface gravity, or the chemical abundances is not expected.

Object 1137 shows a strange $\mathrm{H} \alpha$ line completely filled by wind emission leading to a flat, almost continuum-like profile almost without an indication of the line being present. Therefore, we adjust $\mathrm{H} \beta$ in such way that the calculations do not lead to the rise of extended stellar wind emission wings. The massloss rate derived in this way is then an upper limit. We find a mean metallicity for this star of $1.0 Z_{\odot}$. The derived parameters are consistent with our initial classification as a mid-B Sg.

\subsubsection{Stars of the Keck ESI Data Set}

For the supergiant OB 112-41, the hydrogen Balmer lines $\mathrm{H} \gamma$ and $\mathrm{H} \beta$ are well represented by the models, but the observed red wing of $\mathrm{H} \delta$ is not well fitted. This is possibly due to the presence of $\mathrm{O}$ II lines, formed in the red wing of the line, which are not yet included in our models. He lines are generally well reproduced, except $\mathrm{He}_{\mathrm{I}} \lambda \lambda 5875$ and 6678 . This seems to be a systematic problem also for other stars. In order to reproduce the line widths, a large $v_{\text {turb }}$ is required, which at the same time produces cores deeper than observed. Also, we always produce stronger $\mathrm{He}$ II $\lambda 4686$ than observed. Strong Si Iv lines could require a large turbulence also; however, the weak Si Iv line at $4212 \AA$ appears well reproduced. The effective temperature is well constrained from the $\mathrm{He}_{\mathrm{I}} / \mathrm{He}_{\text {II }}$ ionization balance, as well as from the $\mathrm{O}_{\text {II }} / \mathrm{O}_{\text {III }}$ lines, which are well reproduced.

OB 10-10 shows $\mathrm{H} \beta$ as a $\mathrm{P}$ Cygni profile, as well as a full emission profile in $\mathrm{H} \alpha$, which indicates a strong mass-loss rate;

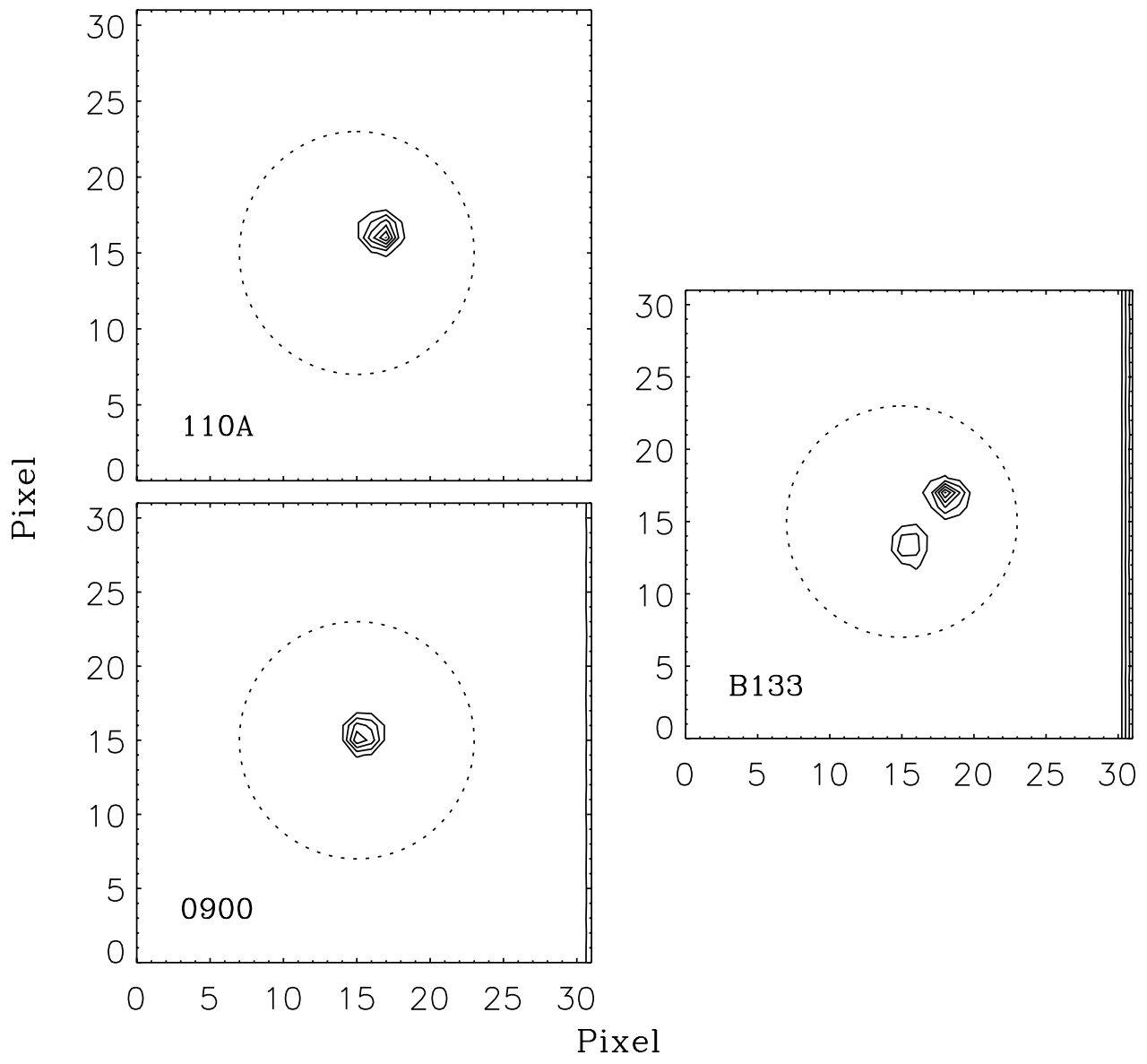

FIG. 7.- - Isocontour plots obtained from HST STIS CCD acquisition images (GO program 7481, PI: Kudritzki) for 110A, B133, and 0900. The dotted circle has a diameter of $1^{\prime \prime}$. Note the presence of two close objects in the location of B133, which cannot be resolved in ground observations. 
the $\dot{M}$ derived to fit both emissions produces too much wind emission in other higher Balmer lines. Along with this high $\dot{M}$, the large value derived for the $\beta$-parameter possibly reflects an unsuitable description of the velocity field in the wind regime or, equivalently, the presence of inhomogeneities in the mass outflow. In some way, OB 10-10 shows the same problems as 110A, but at a lower level. We derive $\alpha$-element abundances leading to a mean metallicity of $1.0 Z_{\odot}$.

The stars UIT 103, UIT 122, and UIT 136 show very similar stellar parameters, although they have different metallicities. Contamination by $\mathrm{H}$ in region emission can be identified in both UIT 122 and UIT 136 by the presence of the emission lines of [S II] $\lambda \lambda 6713,6731$; an $\mathrm{H}$ II H $\alpha$ component is notorious in both stellar spectra also, although for UIT 136 it does not provide a serious problem since the stellar component can be very well resolved. UIT 122 shows a stronger mass-loss rate than the other two stars, which is consistent with its higher metallicity.

UIT 103 shows very weak He II lines, but they still can be used along with $\mathrm{Si}$ lines to determine $T_{\text {eff }}$. The reduced metallicity weakens the $\mathrm{O}$ III line, which prevents the use of the $\mathrm{O}$ II $/ \mathrm{O}_{\text {III }}$ ionization balance to constrain $T_{\text {eff }}$. Nitrogen lines look well reproduced, as well as $\mathrm{O}$ II lines.

For UIT 122 the He II lines appear as weak features but are still useful as a constraint for $T_{\text {eff }}$. As in previous cases, He II $\lambda 4686$ is stronger in the models than observed. Lines from $\mathrm{He}_{\mathrm{I}}, \mathrm{N}$ II, and $\mathrm{O}$ II are well matched.

UIT 136 represents one of the best reproduced spectra, except again for He II $\lambda 4686$. $\mathrm{H} \beta$ shows emission blueward of the line core; the relative displacement is consistent with the observed shift of the $\mathrm{H}$ II emission component relative to the stellar $\mathrm{H} \alpha$ profile.

\subsubsection{Final Considerations}

Several problems were encountered during the analysis work, which we briefly summarize here. Work is in progress to address these issues:

1. The Si IV $\lambda \lambda 4089,4116$ problem: for B0 supergiants we have found that the ionization equilibrium of these lines relative to Si III and the effective temperatures derived accordingly are inconsistent with the values determined from the $\mathrm{He}_{\mathrm{I}} / \mathrm{He}$ II ionization balance, with the derived $T_{\text {eff }}$ being $\sim 1500-2000 \mathrm{~K}$ hotter when using $\mathrm{He}_{\mathrm{I}} / \mathrm{He}$ II lines. This discrepancy is also found in stars with lower luminosities; for example, an analysis of the Galactic O9 V star 10 Lac reveals a difference of $2000 \mathrm{~K}$ between the two $T_{\text {eff }}$ indicators. Given the fact that the He ionization balance has been successfully applied in the analysis of hot spectral types (e.g., Herrero et al. 2002), yielding consistent results when comparing spectral analyses of the same objects observed in different ranges (Repolust et al. 2004, 2005), we have to conclude that the $\mathrm{He}$ I/He II ionization balance is producing the correct results and hence the problem resides in the Si model atom. A possible explanation for the discrepancy follows: the synthetic Si Iv lines produced by our model atom seem to reach their saturation limit at these hotter spectral types. A reduction in $T_{\text {eff }}$ would shift the Si ionization balance toward the lower ionization stage, producing a partial desaturation of the lines. Along with that, a somewhat larger turbulent velocity would be required, which would also contribute to desaturating the lines. However, the introduction of such changes in the parameters would restrain the He lines from being well reproduced. In order to clarify this question, a detailed revision and extension of our Si model is currently under way.

2. The $\mathrm{N}$ III line spectrum problem: in general, we cannot find a good fit of $\mathrm{N}$ II and $\mathrm{N}$ III lines simultaneously. It is well known that the strongest $\mathrm{N}_{\text {III }}$ lines present in the optical spectrum of $\mathrm{OB}$ supergiants are affected by the dielectronic recombination process (Mihalas et al. 1972). This becomes important at earlier spectral types (as is the case for OB 10-3), for which only lines from $\mathrm{C}$ III/C IV and $\mathrm{N}$ III/ $\mathrm{N}$ IV are present in the optical spectra.

3. Helium: there are still several open questions related to modeling the helium spectra of OB stars. Possibly one of the most important ones concerns the reproduction of the He II line at $4686 \AA$. At the present stage, we cannot find any satisfactory solution for this line in our models, in which the synthetic line is systematically stronger than the observed, although all the other He II lines present are generally well reproduced. Three of the observed He I lines (the lines at 4471, 5875, and $6678 \AA$ ) show discrepancies for all the stars. Although the well-known dilution problem of $\mathrm{He}$ I $\lambda 4471$ seems not to be present in some cases, the two other lines, $\mathrm{He}_{\mathrm{I}} \lambda \lambda 5875$ and 6678 , are in all cases deeper and narrower in models than observed.

4. The $\mathrm{C}$ II problem: it is widely accepted that the $\mathrm{C}$ II line at $4267 \AA$ yields unrealistic low abundances when compared with other indicators (for example, $\mathrm{C}$ abundances derived from $\mathrm{F}$ - and G-type stars). Even for B-type dwarf stars in the solar neighborhood, modeling this spectral feature has proved to be a very difficult task, either in LTE or non-LTE (see, e.g., Vrancken et al. 2000 and references therein). It is unclear where the problem resides, since several authors applying different model atoms found similar results. There is not an easy way to detect and to solve this long-standing problem.

\section{DISCUSSION}

The stellar parameters and chemical abundances derived in the analysis are summarized in Tables 7, 8, and 9. The observed blue spectra of B38 and UIT 103 are displayed in Figures 8 and 9, respectively, along with the final FASTWIND spectra calculated with the final parameters.

In general, we find large values of $v_{\text {turb }}$, some of them of the order of the characteristic speed of sound for these stars (around $20 \mathrm{~km} \mathrm{~s}^{-1}$ ); such large turbulent velocities in general produce a very good visual match of the observed spectra. A reduction by $5 \mathrm{~km} \mathrm{~s}^{-1}$ in adopted $v_{\text {turb }}$ would produce changes up to $0.1 \mathrm{dex}$ in abundances, depending on the model itself and the considered line. This does not seem to be a large change but will increase the line-to-line abundance dispersion. As we have quoted before, some of the observed $\mathrm{He}$ I lines require even larger turbulences in order to reproduce their widths, but this produces synthetic lines deeper than the observed. The treatment of the microturbulence still represents the weakest point of the stellar analysis.

Observed and derived photometric quantities are given in Table 10. Massey et al. (1995) concluded that the average reddening of M33 is $E(B-V)=0.13 \mathrm{mag}$, with OB associations ranging from 0.08 to $0.33 \mathrm{mag}$. The reddening from our spectroscopic work is somewhat lower, $E(B-V)=0.08 \mathrm{mag}$, but still compatible with the previous value within the uncertainties. We also note that our targets were chosen as the brightest, and hence least reddening-affected, stars. For those stars that are members of the OB associations in the survey of Massey et al. (1995), we find good agreement between mean photometric reddenings and our individual spectroscopic values. For example, Massey et al. (1995) reported a mean color excess of $0.13 \pm$ $0.01 \mathrm{mag}$ for OB 21, while we found $0.10 \mathrm{mag}$ for OB 21-108. It is important to note, however, that from the work on Galactic OB associations of Garmany \& Stencel (1992) the standard deviation in reddening within a given $\mathrm{OB}$ association is $\sim 0.25 \mathrm{mag}$. This is very well illustrated by the cases of $\mathrm{OB} 10-3$ and $\mathrm{OB} 10-10$, 
TABLE 7

Derived Stellar Parameters and Chemical Abundances for the Stars in Common with Monteverde et al. (2000)

\begin{tabular}{|c|c|c|c|c|}
\hline Properties & B38 & $110 \mathrm{~A}$ & B133 & 1054 \\
\hline Alternate ID $^{\mathrm{a}} \ldots \ldots \ldots$ & OB 21-108, UIT 030 & 0785 & 0515, UIT 107 & UIT 215 \\
\hline $\mathrm{SpT}$ & B1 Ia & $\mathrm{B} 1 \mathrm{Ia}^{+}$ & B1.5 Ia & $\mathrm{B} 2 \mathrm{Ib}$ \\
\hline$\rho / \rho_{0}{ }^{\mathrm{b}}$. & 0.52 & 0.70 & 0.56 & 0.04 \\
\hline 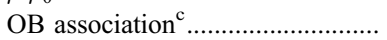 & OB 21 & OB 110 & OB 31 & OB 142 \\
\hline$T_{\text {eff }}(\mathrm{kK})$ & $22.0 \pm 0.5$ & $21.0 \pm 1.0$ & $21.5 \pm 1.0$ & $20.5 \pm 1.0$ \\
\hline $\log g(\operatorname{cgs})$ & $2.60 \pm 0.10$ & $2.66 \pm 0.15$ & $2.70 \pm 0.10$ & $2.60 \pm 0.10$ \\
\hline$v_{\infty}\left(\mathrm{km} \mathrm{s}^{-1}\right)$ & 730 & 750 & 900 & 800 \\
\hline$\dot{M}\left(10^{-6} M_{\odot} \mathrm{yr}^{-1}\right) \ldots \ldots$ & $1.06 \pm 0.24$ & $10.6 \pm 0.61$ & $0.80 \pm 0.12$ & $0.68 \pm 0.15$ \\
\hline$R\left(R_{\odot}\right) \ldots \ldots \ldots$ & $53.5 \pm 1.5$ & $80.1 \pm 2.0$ & $60.0 \pm 2.0$ & $85.0 \pm 1.5$ \\
\hline$\beta$ & $2.35 \pm 0.15$ & $3.00 \pm 0.10$ & $3.00 \pm 0.15$ & $2.50 \pm 0.20$ \\
\hline$Y_{\mathrm{He}}$ & 0.10 & 0.20 & 0.20 & 0.10 \\
\hline$v_{\text {turb }}\left(\mathrm{km} \mathrm{s}^{-1}\right) \ldots \ldots$ & $17 \pm 3$ & $15 \pm 4$ & $15 \pm 3$ & $9 \pm 3$ \\
\hline$v \sin i\left(\mathrm{~km} \mathrm{~s}^{-1}\right) \ldots \ldots$ & $<60$ & $<60$ & $<70$ & $<65$ \\
\hline$\epsilon_{\mathrm{C}}$ & $7.45 \pm 0.09( \pm 0.11)$ & $7.55 \pm 0.15( \pm 0.22)$ & $8.00 \pm 0.09$ & $7.45 \pm 0.14$ \\
\hline$\epsilon_{\mathrm{N}} \ldots \ldots \ldots$ & $8.15 \pm 0.17( \pm 0.12)$ & $8.40 \pm 0.20( \pm 0.20)$ & $8.10 \pm 0.16$ & $8.10 \pm 0.15$ \\
\hline$\epsilon_{\mathrm{O}} \ldots \ldots \ldots$ & $8.50 \pm 0.10( \pm 0.10)$ & $8.50 \pm 0.17( \pm 0.25)$ & $8.30 \pm 0.17$ & $8.90 \pm 0.18$ \\
\hline$\epsilon_{\mathrm{Mg}}$ & $7.30 \pm 0.07( \pm 0.07)$ & $7.40 \pm 0.10( \pm 0.19)$ & $7.75 \pm 0.10$ & $7.50 \pm 0.12$ \\
\hline$\epsilon_{\mathrm{S}}$ & $7.35 \pm 0.08( \pm 0.11)$ & $7.35 \pm 0.12( \pm 0.19)$ & $7.45 \pm 0.15$ & $7.40 \pm 0.17$ \\
\hline$Z^{\alpha}\left(Z_{\odot}\right)$ & 0.60 & 0.60 & 0.50 & 0.80 \\
\hline$[\mathrm{M} / \mathrm{H}](\mathrm{dex}) \ldots \ldots \ldots \ldots$ & -0.22 & -0.22 & -0.30 & -0.10 \\
\hline $\log \left(L / L_{\odot}\right)(\operatorname{cgs}) \ldots \ldots$ & $5.77 \pm 0.06$ & $6.50 \pm 0.10$ & $6.17 \pm 0.11$ & $6.06 \pm 0.10$ \\
\hline$M^{\text {spec }}\left(M_{\odot}\right) \ldots \ldots$ & $38 \pm 11$ & $98 \pm 39$ & $60 \pm 18$ & $96 \pm 26$ \\
\hline $\log D_{\text {mod }} \ldots \ldots$ & $28.90 \pm 0.07$ & $29.45 \pm 0.09$ & $28.55 \pm 0.10$ & $28.50 \pm 0.21$ \\
\hline
\end{tabular}

Notes.-Systematic uncertainties for B133 and 1054 are the same as the ones quoted for 110A and B38, respectively. The metal abundances are expressed as $\epsilon_{\mathrm{A}}=12+\log (\mathrm{A} / \mathrm{H})$, while $[\mathrm{M} / \mathrm{H}]=\log (\mathrm{M} / \mathrm{H})_{*}-\log (\mathrm{M} / \mathrm{H})_{\odot}$. Adopted quantities are quoted in italics, without uncertainties. The modified wind momentum is defined as $D_{\text {mod }}=\dot{M} v_{\infty} R^{1 / 2}$.

${ }^{a}$ Ivanov et al. (1993); Massey et al. (1995, 1996); Humphreys \& Sandage (1980).

${ }^{\mathrm{b}}$ Deprojected radial distance in units of the Holmberg radius. Adopted quantities: $\rho_{0}=28.77, i=54^{\circ}$, and P.A. $=22^{\circ}$.

c OB identification from Humphreys \& Sandage (1980).

for which we found a color excess of 0.06 and $0.24 \mathrm{mag}$, respectively, again in agreement with the 0.20 mag quoted by Massey et al. (1995).

Figure 10 shows the comparison of derived stellar parameters and chemical abundances for the stars in common with Monteverde et al. (2000). Note that these authors used planeparallel hydrostatic unblanketed models in their analysis. In general, our analyses yield larger gravities. The reasons for the systematic differences in gravities have been discussed in the chapters before. An additional effect, at least for stars with low wind densities, is the consideration of the photospheric line radiation pressure in blanketed models, which reduces the effective gravity. As a consequence, a larger gravity is needed in blanketed models to reproduce the $\mathrm{H}$ line profiles. Despite the differences in the stellar parameters, the chemical abundances, in particular for oxygen, are in good agreement.

\subsection{Evolutionary Status}

In this section we compare the derived stellar parameters and chemical abundances with the predictions of state-of-the-art stellar evolutionary models taken from Meynet \& Maeder (2003) for $z=0.02$ and from Meynet \& Maeder (2005) for $z=0.008$. Of the set of published models we only consider those with initial equatorial rotational velocity of $300 \mathrm{~km} \mathrm{~s}^{-1}$.

Figure 11 shows the comparison of spectroscopic effective temperatures and surface gravities with model predictions for several initial stellar masses. As can be seen, our program stars appear to be located between the 20 and $60 M_{\odot}$ tracks (almost independent of the metallicity of the models). Note that we choose this representation of stellar evolution (equivalent to the HRD) because the considered parameters do not depend on photometric measurements and assumptions on the distance (unlike luminosities in the HRD). As expected, the stars have evolved beyond the theoretical turnover of the MS. In Figure 12 we present the comparison of surface gravities and stellar radii. Note that the latter depend on photometric magnitudes; thus, the comparison to model predictions can be hampered by the presence of unresolved companions. Indeed, two of the stars are clearly above the predictions of a $60 M_{\odot}$ track. A third one is in marginal disagreement. From higher to lower stellar radius, these three stars are 1054,110A, and B133, respectively. As we have seen previously, both 1054 and B133 have very close confirmed companions, which affects the available photometry. The case of $110 \mathrm{~A}$ is somewhat ambiguous because we did not find an indication of a companion at the resolution of HST STIS acquisition images, which, of course, does not completely rule out the presence of such a companion. The location of the other stars in the sample in this diagram is consistent with the previous figure (within the predictions for 20 and $60 M_{\odot}$ ).

Concerning the comparison of evolutionary and spectroscopic masses, the agreement is good within the uncertainties, except for 110A, 1054, and B133, for which we found larger masses than predicted (see previous discussion on stellar radius).

The problem of the radius of $110 \mathrm{~A}$ becomes also evident in Figure 13, a classic representation of a theoretical HRD, where we have included the Humphreys-Davidson limit as a dotted line. Note that once more three stars appear clearly separated from the other "normal" B-type supergiant group; from higher to lower luminosities they are 110A (above the HD limit), 1054, and B133. Our results locate 110A in a forbidden region populated by LBV stars only. However, 110A does not show the typical emissionline spectra of LBV stars. Even more puzzling is the fact that its 
TABLE 8

Stellar Parameters and Chemical Abundances for New WHT ISIS Targets

\begin{tabular}{|c|c|c|}
\hline Properties & 0900 & 1137 \\
\hline Alternate ID $^{\mathrm{a}} \ldots \ldots \ldots \ldots \ldots \ldots$ & $\mathrm{B} 217 \mathrm{a}$ & B354, UIT 235 \\
\hline SpT & B0.5-B1 Ia & B2.5 Ib-B3 Ia \\
\hline$\rho^{\mathrm{p}} \rho_{0}^{\mathrm{b}}$ & 0.12 & 0.20 \\
\hline $\mathrm{OB}$ association ${ }^{\mathrm{c}} \ldots \ldots \ldots \ldots$ & OB 48 & OB 3 \\
\hline 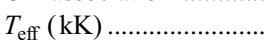 & $23.0 \pm 1.0$ & $20.0 \pm 1.0$ \\
\hline $\log g(\operatorname{cgs}) \ldots$ & $2.62 \pm 0.15$ & $2.50 \pm 0.10$ \\
\hline$v_{\infty}\left(\mathrm{km} \mathrm{s}^{-1}\right) \ldots \ldots \ldots \ldots \ldots$ & 950 & 750 \\
\hline$\dot{M}\left(10^{-6} M_{\odot} \mathrm{yr}^{-1}\right) \ldots \ldots$ & $<2.24$ & $0.69 \pm 0.15$ \\
\hline$R\left(R_{\odot}\right) \ldots$ & $47.0 \pm 1.0$ & $56.5 \pm 1.5$ \\
\hline$\beta$ & 1.00 & $1.50 \pm 0.30$ \\
\hline 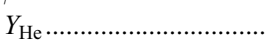 & 0.10 & 0.10 \\
\hline$v_{\text {turb }}\left(\mathrm{km} \mathrm{s}^{-1}\right) \ldots \ldots \ldots \ldots$ & 15 & $8 \pm 2$ \\
\hline$v \sin i\left(\mathrm{~km} \mathrm{~s}^{-1}\right) \ldots \ldots \ldots$ & $<70$ & $<60$ \\
\hline$\epsilon_{\mathrm{C}} \cdots \ldots$ & $7.45 \pm 0.16( \pm 0.23)$ & $8.00 \pm 0.14( \pm 0.09)$ \\
\hline$\epsilon_{\mathrm{N}}$ & $8.30 \pm 0.19( \pm 0.21)$ & $8.50 \pm 0.16( \pm 0.14)$ \\
\hline$\epsilon_{\mathrm{O}}$ & $8.75 \pm 0.22( \pm 0.14)$ & $8.70 \pm 0.15( \pm 0.10)$ \\
\hline$\epsilon_{\mathrm{Mg}} \ldots \ldots \ldots$ & $7.50 \pm 0.14( \pm 0.21)$ & $7.60 \pm 0.12( \pm 0.08)$ \\
\hline$\epsilon_{\mathrm{S} 1} \ldots \ldots \ldots \ldots \ldots \ldots \ldots \ldots \ldots \ldots \ldots$ & $7.45 \pm 0.17( \pm 0.21)$ & $7.45 \pm 0.19( \pm 0.12)$ \\
\hline$Z^{\alpha}\left(Z_{\odot}\right)$ & 0.80 & 0.80 \\
\hline$[\mathrm{M} / \mathrm{H}](\mathrm{dex}) \ldots \ldots \ldots \ldots \ldots$ & -0.10 & -0.10 \\
\hline $\log \left(L / L_{\odot}\right) \ldots \ldots$ & $5.75 \pm 0.12$ & $5.55 \pm 0.11$ \\
\hline$M^{\text {spec }}\left(M_{\odot}\right) \ldots \ldots \ldots \ldots \ldots$ & $31 \pm 12$ & $34 \pm 10$ \\
\hline $\log D_{\bmod } \ldots \ldots \ldots \ldots$ & $28.94 \pm 0.16$ & $28.39 \pm 0.12$ \\
\hline
\end{tabular}

Notes.-The metal abundances are expressed as $\epsilon_{\mathrm{A}}=12+\log (\mathrm{A} / \mathrm{H})$, while $[\mathrm{M} / \mathrm{H}]=\log (\mathrm{M} / \mathrm{H})_{*}-\log (\mathrm{M} / \mathrm{H})_{\ldots}$. Adopted quantities are quoted in italics, without uncertainties. The modified wind momentum is defined as $D_{\text {mod }}=\dot{M} v_{\infty} R^{1 / 2}$.

a Ivanov et al. (1993); Massey et al. (1995, 1996); Humphreys \& Sandage (1980).

b Deprojected radial distance in units of the Holmberg radius. Adopted quantities: $\rho_{0}=28.77, i=54^{\circ}$, and P.A. $=22^{\circ}$.

${ }^{c}$ OB identification from Humphreys \& Sandage (1980). luminosity puts 110A among the most luminous LBVs known. We cannot rule out that $110 \mathrm{~A}$ is a completely unusual case. However, there are other possibilities. The star could indeed be a compact group of stars, although then it is unclear why no indication of multiplicity is given by the optical spectrum and the STIS acquisition images. The stellar parameters could be affected by the lack of some physical process not considered in our models (most likely the presence of inhomogeneities in the wind of the star). This raises, of course, the question of why we encounter such a problem for only this particular star.

The evolutionary status of the other stars is, obviously, normal post-MS evolution either toward the red supergiant phase or back from the red giant branch. The comparison of the stellar evolution and observed surface N/O ratio (by number) shown in Figure 14 indicates consistency with evolution from the MS toward the red phase (all the ratios are well below the large increase produced by the first dredge-up in the red supergiant phase). The stars are all located between the 20 and $40 M_{\odot}$ tracks. Although lower initial rotational velocities would produce lower enrichments, the results are also consistent with a somewhat less efficient mixing mechanism (note that most likely the stars in our sample were born with different rotational velocities). This conclusion is in agreement with other recent studies of B-type supergiant stars like those of Urbaneja et al. (2005) in NGC 300 and Trundle et al. (2004) and Trundle \& Lennon (2005) in the SMC and of A-type supergiants in nearby galaxies (Przybilla 2002). We conclude that there is a good agreement between the recent evolutionary models and the stellar parameters and chemical abundances derived.

\subsection{Radial Abundance Gradients in the Disk of M33}

The first quantitative determination of the stellar oxygen abundance gradient in M33 was carried out by Monteverde et al.

TABLE 9

Stellar Parameters and Chemical Abundances of Keck ESI Sample

\begin{tabular}{|c|c|c|c|c|c|}
\hline Properties & OB $112-41$ & OB $10-10$ & UIT 136 & UIT 122 & UIT 103 \\
\hline Alternate $\mathrm{ID}^{\mathrm{a}}$. & B287 & B242, UIT 181 & B157 & & \\
\hline SpT & B0 Ia & $\mathrm{B} 1 \mathrm{Ia}^{+}$ & B0.5 Ia & B0.5 Ia & B0.7 Ia \\
\hline$\rho / \rho_{0}^{\mathrm{b}}$ & 0.74 & 0.31 & 1.06 & 0.50 & 1.14 \\
\hline OB association ${ }^{\mathrm{c}} \ldots \ldots \ldots \ldots \ldots \ldots$ & OB 112 & OB 10 & OB 140 & $\ldots$ & $\ldots$ \\
\hline$T_{\mathrm{eff}}(\mathrm{kK})$ & $26.0 \pm 0.5$ & $22.0 \pm 0.5$ & $24.0 \pm 0.5$ & $24.0 \pm 0.5$ & $23.7 \pm 0.5$ \\
\hline $\log g(\mathrm{cgs}) \ldots \ldots \ldots$ & $2.79 \pm 0.10$ & $2.70 \pm 0.10$ & $2.60 \pm 0.10$ & $2.77 \pm 0.10$ & $2.65 \pm 0.10$ \\
\hline 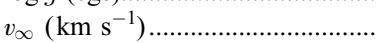 & 1500 & 900 & 1500 & 1500 & 1200 \\
\hline$\dot{M}\left(10^{-6} M_{\odot} \mathrm{yr}^{-1}\right) \ldots \ldots \ldots \ldots \ldots \ldots \ldots$ & $2.09 \pm 0.41$ & $2.20 \pm 0.15$ & $2.11 \pm 0.36$ & $2.40 \pm 0.30$ & $2.04 \pm 0.27$ \\
\hline$R\left(R_{\odot}\right) \ldots \ldots$ & $32.8 \pm 1.0$ & $51.0 \pm 1.0$ & $39.0 \pm 2.0$ & $38.8 \pm 1.7$ & $40.1 \pm 2.0$ \\
\hline$\beta$ & $1.35 \pm 0.20$ & $4.00 \pm 0.10$ & $1.50 \pm 0.15$ & $1.80 \pm 0.15$ & $1.50 \pm 0.15$ \\
\hline 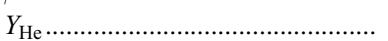 & 0.10 & 0.16 & 0.10 & 0.10 & 0.08 \\
\hline 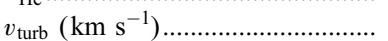 & $17 \pm 3$ & $17 \pm 3$ & $17 \pm 3$ & $17 \pm 3$ & $12 \pm 2$ \\
\hline$v \sin i\left(\mathrm{~km} \mathrm{~s}^{-1}\right) \ldots \ldots \ldots \ldots \ldots \ldots$ & $<65$ & $<50$ & $<70$ & $<70$ & $<50$ \\
\hline$\epsilon_{\mathrm{C}}$ & $7.65 \pm 0.07( \pm 0.08)$ & $7.35 \pm 0.11( \pm 0.11)$ & $7.15 \pm 0.08( \pm 0.08)$ & $7.80 \pm 0.08$ & $6.70 \pm 0.07$ \\
\hline$\epsilon_{\mathrm{N}}$ & $8.00 \pm 0.10( \pm 0.10)$ & $8.55 \pm 0.09( \pm 0.12)$ & $7.70 \pm 0.09( \pm 0.10)$ & $8.10 \pm 0.12$ & $7.90 \pm 0.10$ \\
\hline$\epsilon_{\mathrm{O}}$ & $8.60 \pm 0.06( \pm 0.09)$ & $8.65 \pm 0.12( \pm 0.10)$ & $8.40 \pm 0.09( \pm 0.09)$ & $8.50 \pm 0.06$ & $8.20 \pm 0.13$ \\
\hline$\epsilon_{\mathrm{Mg}}$ & $7.75 \pm 0.10( \pm 0.07)$ & $7.60 \pm 0.08( \pm 0.07)$ & $7.35 \pm 0.07( \pm 0.07)$ & $\ldots$ & $7.10 \pm 0.08$ \\
\hline$\epsilon_{\mathrm{Si}}$ & $7.75 \pm 0.15( \pm 0.10)$ & $7.60 \pm 0.12( \pm 0.11)$ & $7.35 \pm 0.14( \pm 0.11)$ & $7.45 \pm 0.13$ & $7.20 \pm 0.13$ \\
\hline$Z^{\alpha}\left(Z_{\odot}\right)$ & 1.00 & 0.90 & 0.50 & 0.60 & 0.30 \\
\hline 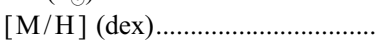 & 0.00 & -0.05 & -0.30 & -0.22 & -0.52 \\
\hline $\log \left(L / L_{\odot}\right)(\operatorname{cgs}) \ldots \ldots$ & $5.68 \pm 0.07$ & $5.73 \pm 0.06$ & $5.65 \pm 0.08$ & $5.66 \pm 0.07$ & $5.68 \pm 0.08$ \\
\hline$M^{\mathrm{spec}}\left(M_{\odot}\right)$ & $22 \pm 6$ & $44 \pm 12$ & $20 \pm 7$ & $30 \pm 9$ & $24 \pm 8$ \\
\hline $\log D_{\bmod } \ldots \ldots \ldots \ldots$ & $29.05 \pm 0.17$ & $28.95 \pm 0.12$ & $29.10 \pm 0.13$ & $29.15 \pm 0.11$ & $28.99 \pm 0.11$ \\
\hline
\end{tabular}

Notes.-Systematic uncertainties for UIT 122 and UIT 103 are the same as the ones quoted for UIT 136. The metal abundances are expressed as $\epsilon_{\mathrm{A}}=12+\log (\mathrm{A} / \mathrm{H})$, while $[\mathrm{M} / \mathrm{H}]=\log (\mathrm{M} / \mathrm{H})_{*}-\log (\mathrm{M} / \mathrm{H})_{\odot}$. Adopted quantities are quoted in italics, without uncertainties. The modified wind momentum is defined as $D_{\bmod }=\dot{M} v_{\infty} R^{1 / 2}$

${ }^{a}$ Ivanov et al. (1993); Massey et al. (1995, 1996); Humphreys \& Sandage (1980).

b Deprojected radial distance in units of the Holmberg radius. Adopted quantities: $\rho_{0}=28$ ' $77, i=54^{\circ}$, and P.A. $=22^{\circ}$.

c OB identification from Humphreys \& Sandage (1980). 

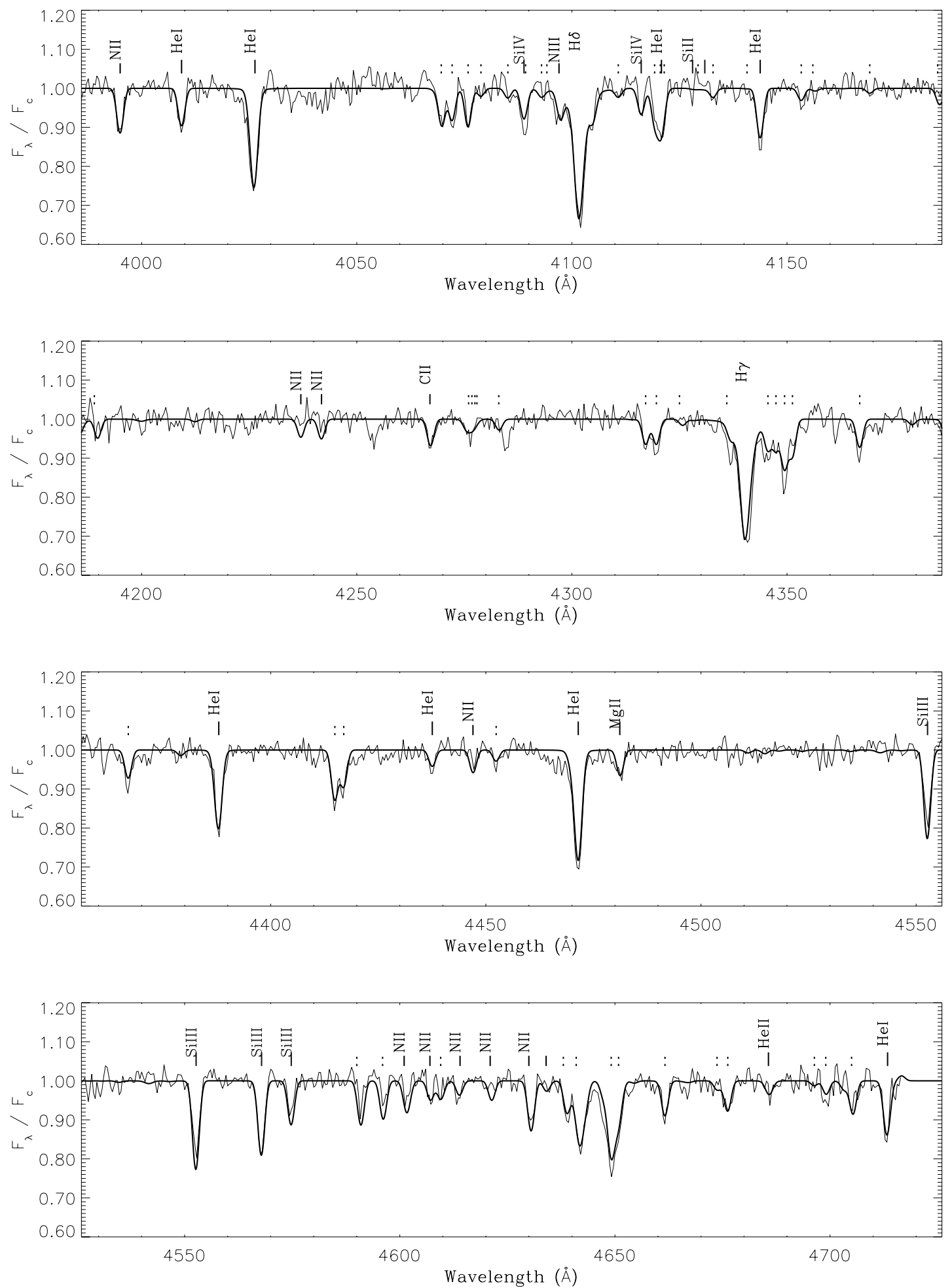

FIG. 8.-Observed (WHT ISIS) blue spectrum of B38 and the synthetic one that results from our analysis. An identification of prominent features is also provided. Note that dotted lines without labels correspond to $\mathrm{O}$ II lines. 

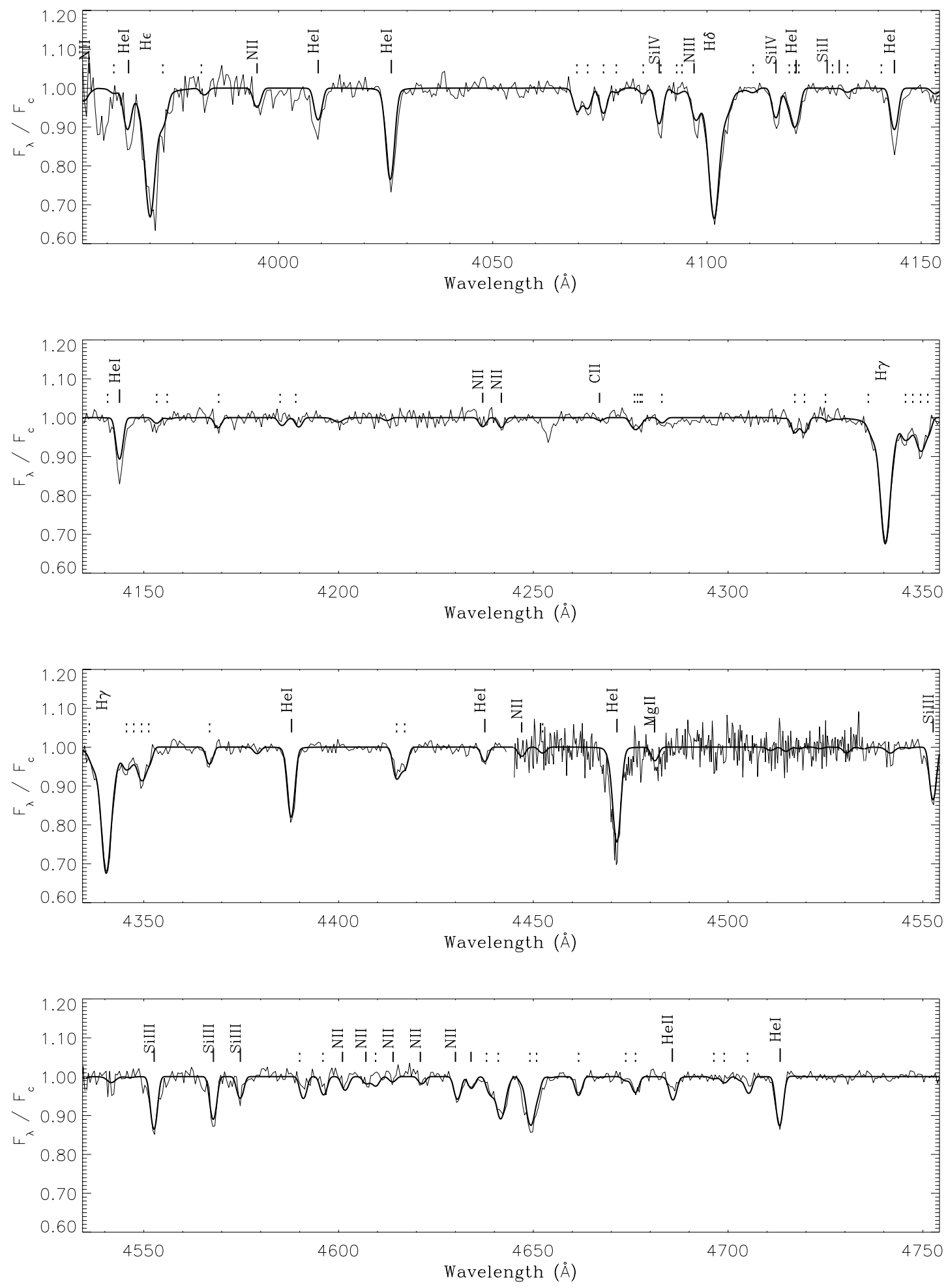

FIG. 9. - Same as Fig. 8, but for the observed (Keck ESI) blue spectrum of UIT 103, showing the excellent agreement in a much wider spectral window. The $~ 4450-$ $4540 \AA$ wavelength range corresponds to WHT ISIS observations (note the reduction of the S/N). 

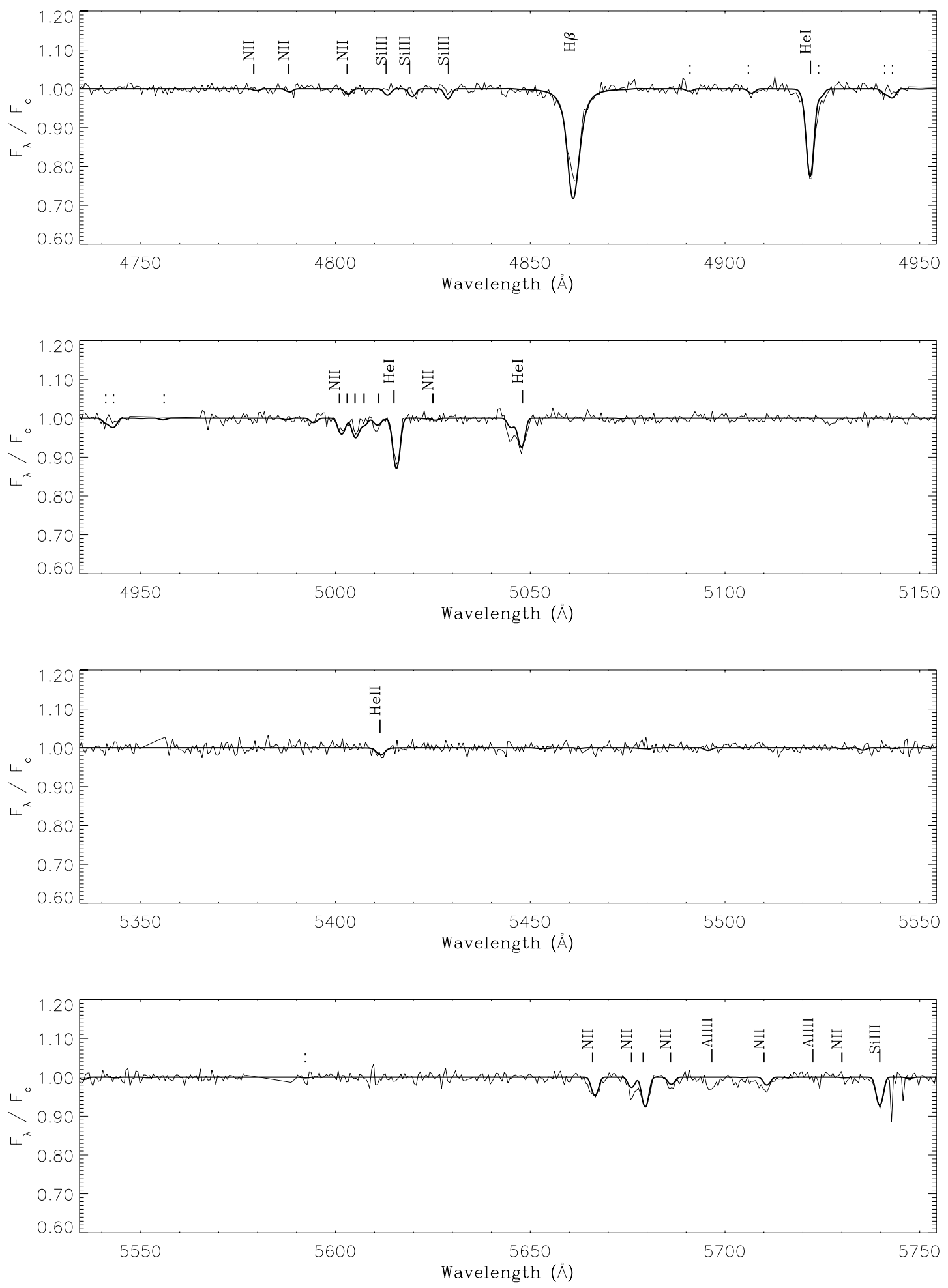

FIG. 9.-Continued 
TABLE 10

Observed and Synthetic Photometric Magnitudes

\begin{tabular}{|c|c|c|c|c|c|c|c|c|}
\hline \multirow[b]{2}{*}{ StAR ID } & \multicolumn{3}{|c|}{ OBSERVED } & \multicolumn{4}{|c|}{ Synthetic } & \multirow[b]{2}{*}{$E(B-V)$} \\
\hline & $V$ & $B-V$ & References & $M_{V}$ & $M_{B}-M_{V}$ & $\mathrm{BC}$ & $M^{\text {bol }}$ & \\
\hline OB 112-41 .................. & 18.01 & -0.15 & Massey et al. (1995) & -6.67 & -0.19 & -2.47 & -9.14 & 0.04 \\
\hline 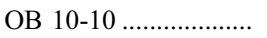 & 17.30 & -0.12 & Massey et al. (1996) & -7.42 & -0.18 & -2.17 & -9.59 & 0.06 \\
\hline 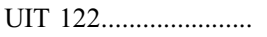 & 17.81 & -0.09 & Massey et al. (1996) & -7.06 & -0.18 & -2.34 & -9.40 & 0.09 \\
\hline 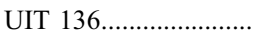 & 17.98 & -0.15 & Massey et al. (1996) & -7.05 & -0.17 & -2.34 & -9.39 & 0.02 \\
\hline 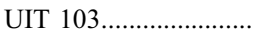 & 17.88 & -0.10 & Massey et al. (1996) & -7.12 & -0.18 & -2.32 & -9.44 & 0.08 \\
\hline 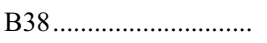 & 17.30 & -0.07 & Massey et al. (1996) & -7.57 & -0.17 & -2.11 & -9.68 & 0.10 \\
\hline 110A & 16.10 & 0.10 & Ivanov et al. (1993) & -9.02 & -0.09 & -2.50 & -11.52 & 0.19 \\
\hline 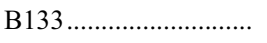 & 16.69 & -0.08 & Massey et al. (1996) & -8.11 & -0.17 & -2.57 & -10.68 & 0.09 \\
\hline 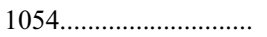 & 16.49 & -0.22 & Massey et al. (1996) & -8.47 & -0.17 & -1.94 & -10.41 & -0.05 \\
\hline 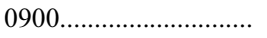 & 17.40 & -0.10 & Ivanov et al. (1993) & -7.41 & -0.18 & -2.22 & -9.63 & 0.08 \\
\hline 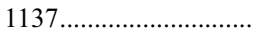 & 17.07 & -0.15 & Massey et al. (1996) & -7.52 & -0.16 & -1.60 & -9.12 & 0.01 \\
\hline
\end{tabular}

Note.-We adopted a distance modulus $\mu=24.56 \pm 0.10$ (Freedman et al. 2001) and $R_{v}=3.1$ for estimating the extinction from derived reddenings.

(2000), who analyzed a sample of four B-type supergiants (1054, 110A, B133, and B38) using non-LTE plane-parallel unblanketed and hydrostatic models. For this sample, ranging from almost the center of the galaxy out to a (deprojected) distance of $0.7 \rho_{0}$, Monteverde et al. (2000) found steep stellar gradients for $\mathrm{O}$ and $\mathrm{Si}$. As we have seen for the stars in common, we obtain $\mathrm{O}$ abundances that are in good agreement with those from Monteverde et al. (2000), despite some differences in the stellar parameters.
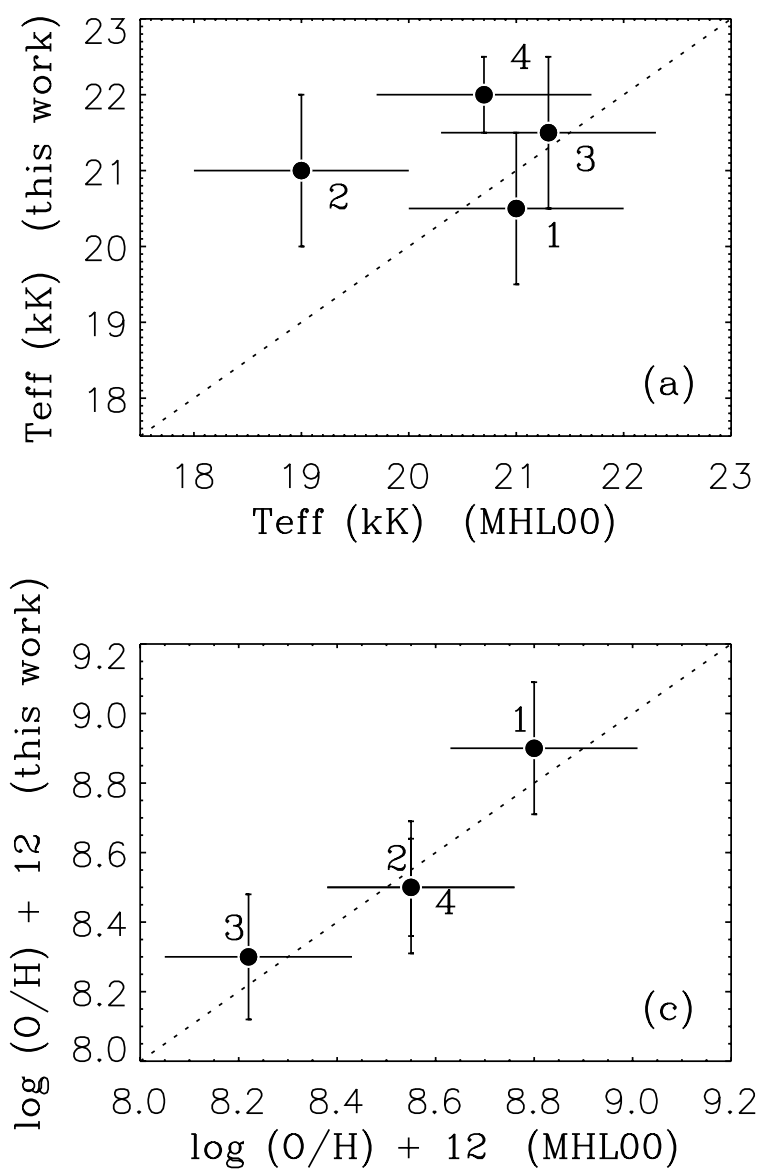

However, considering our full sample with more stars, we find a shallower gradient out to a galactocentric distance of $\sim 1.1 \rho_{0}$. We obtain a negative $O$ gradient of $0.015 \pm 0.005 \mathrm{dex} \operatorname{arcmin}^{-1}$ (or $0.42 \pm 0.14 \mathrm{dex} / \rho_{0}$ ).

Figure 15 shows the comparison of our stellar oxygen abundances with the ISM oxygen abundances from Vílchez et al. (1988), obtained by means of direct measurements of the electron temperature of the nebulae [from here on we refer to this
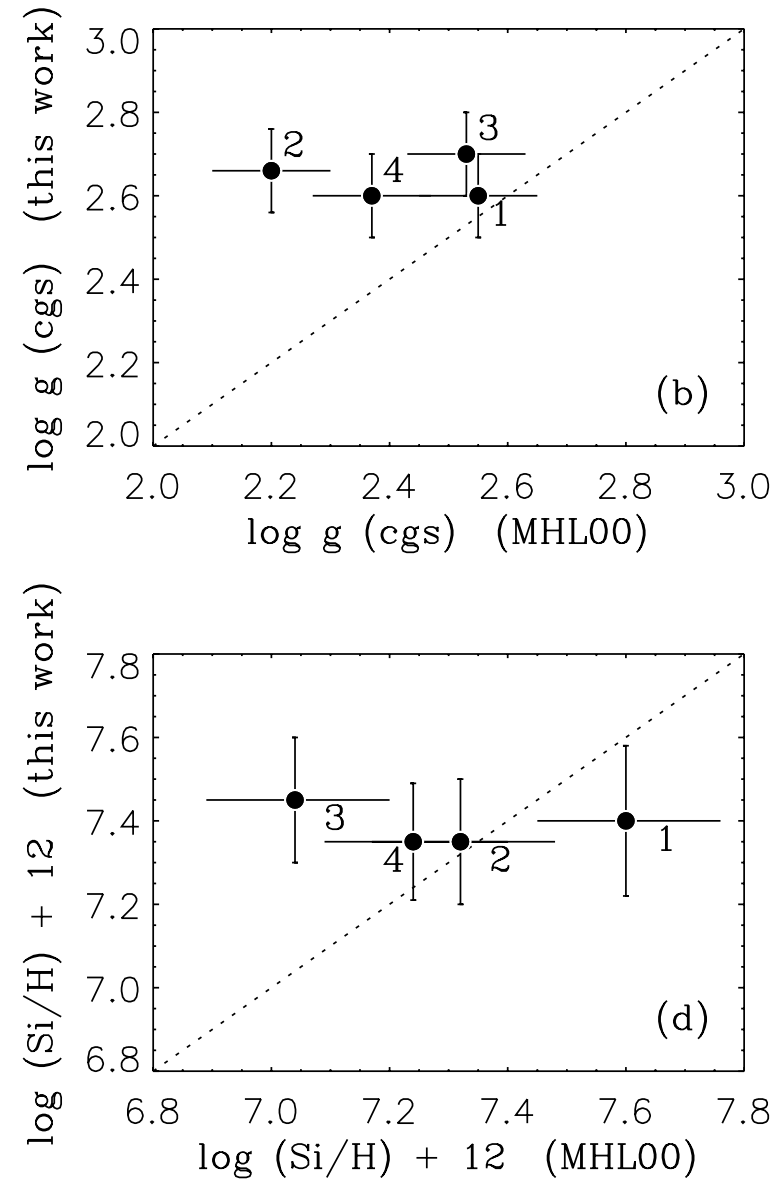

FIG. 10.-Comparison of the stellar parameters and chemical abundances (O and Si) of the stars in common with Monteverde et al. (2000). Star identification:

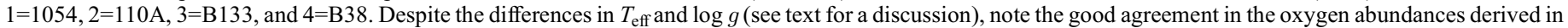
both works. Dotted lines mark the 1:1 relationship loci. 


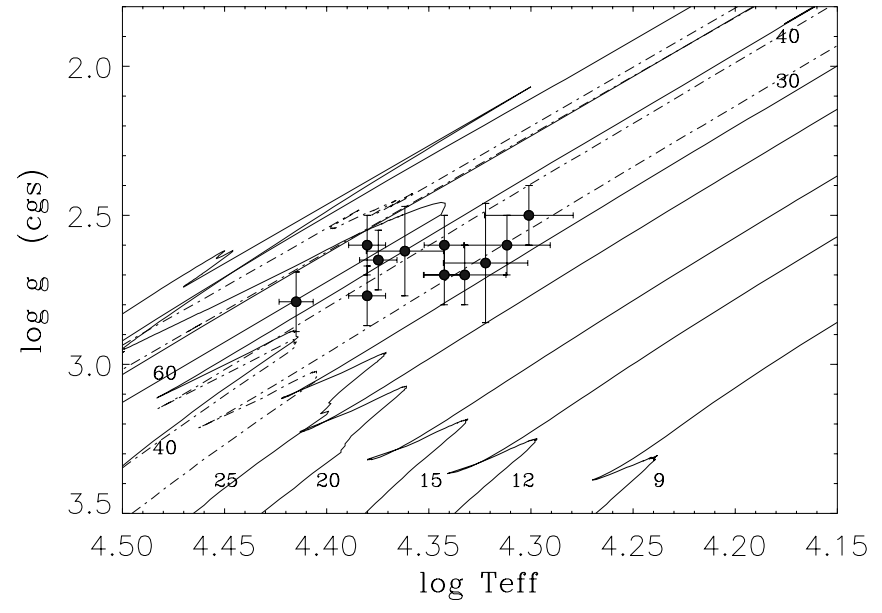

FIG. 11.-Derived $T_{\text {eff }}-\log g$ compared with the predictions of stellar evolutionary models from Meynet \& Maeder (2003) for solar metallicity (tracks for zero-age main-sequence masses $9,12,15,20,25,40$, and $60 M_{\odot}$ are displayed as solid lines) and from Meynet $\&$ Maeder (2005) for $z=0.008$ (only tracks for 30 and $40 M_{\odot}$ are shown as dot-dashed lines and are labeled in the upper right corner).

kind of abundance as $\left.\operatorname{ISM}\left(T_{e}\right)\right]$. Although the uncertainties of the stellar data are large, there is a very good agreement between both sources, even for the inner parts of the galaxy. Vílchez et al. (1988) suggested the possibility of a radial gradient with two different slopes, a shallow gradient for $\rho / \rho_{0}<0.3$ and another steeper one for $\rho / \rho_{0}>0.3$; however, this interpretation was strongly dependent on the central object, for which the authors could not detect the auroral line [O III] $\lambda 4363$ and therefore used a combination of theoretical results from photoionization models to estimate $T_{e}$. In the case of the stellar data, there is no significant difference if we discard 1054 in the weighted leastsquares fit, for which we then obtain a negative slope of $0.012 \pm$ $0.006 \mathrm{dex} \operatorname{arcmin}^{-1}$ (or $0.36 \pm 0.16 \mathrm{dex} / \rho_{0}$ ). We cannot completely rule out a two-slope solution, but our results are consistent with a single-slope gradient.

Vila-Costas \& Edmunds (1992), in their extensive study of the relation between abundance gradients and other physical properties of a large sample of spiral galaxies, made a compilation of M33 H II region data. For M33, the authors find an oxygen abundance gradient of -0.021 dex $\operatorname{arcmin}^{-1}$, with a central oxygen abundance of 8.94 dex, considering 37 regions from different

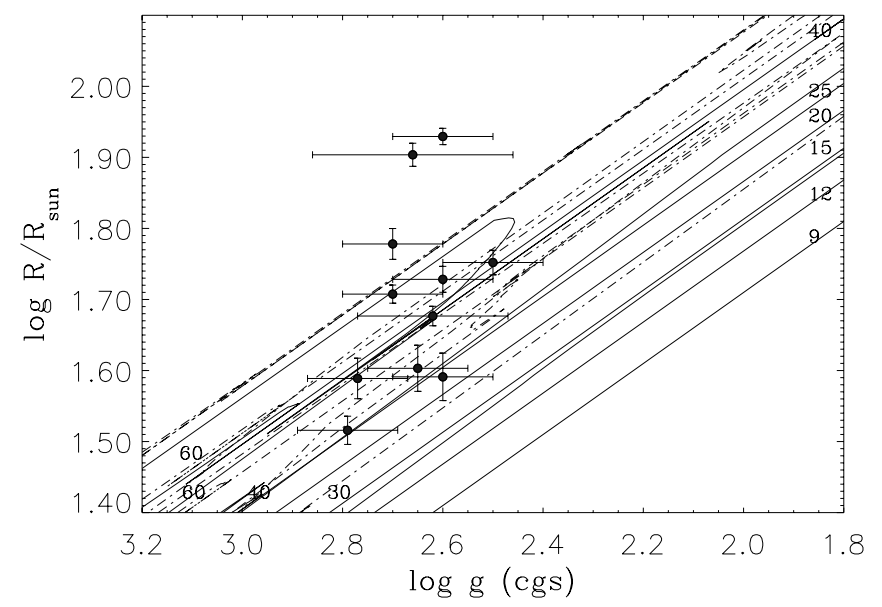

FIG. 12.-Same as Fig. 11, but for surface gravities and stellar radii. Note that we have included a $60 M_{\odot}$ track for $z=0.008$. The three stars above the tracks are, from top to the bottom, 1054, 110A, and B133.

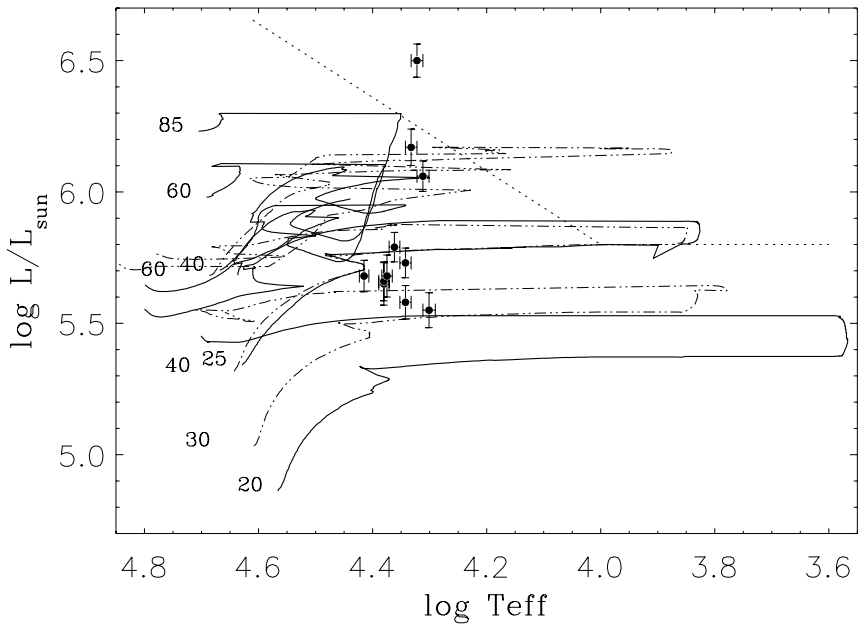

FIG. 13.-Location of the stars in a theoretical HRD. The star well above the HD limit (Humphreys \& Davidson 1979; dotted line) is 110A, while the two other most luminous objects are 1054 and B133. Tracks for 30, 40, and $60 M_{\odot}$ are shown as double-dot-dashed lines for $z=0.008$, while models for 20, 25, 40,60 , and 80 are displayed as solid lines for $z=0.02$. As in previous figures, the evolutionary tracks are from Meynet $\&$ Maeder (2003) for $z=0.02$ and from Meynet \& Maeder (2005) for $z=0.008$.

sources (mostly $\mathrm{O} / \mathrm{H}$ abundance based on statistical methods). This gradient is also consistent with the one we obtain from the B-type supergiants, although the central value is somewhat higher.

Recently, Magrini et al. (2004) determined the oxygen abundances of a sample of planetary nebulae ( $\mathrm{PNe}$ ) in M33. We have used the coordinates reported in Magrini et al. (2001), along with the P.A. and inclination angle taken from Vila-Costas \& Edmunds (1992), to determine the deprojected distance to the center of M33. The comparison of PN O abundances with the B-type supergiant $\mathrm{O}$ abundances is displayed in Figure 16. Despite the dispersion in the $\mathrm{PN}$ results ${ }^{4}$ and the large uncertainties, the agreement is good, in particular if we consider only the four PNe with welldetermined electron temperatures (identified as numbers 28,60 , 96, and 101 in the cited reference and represented by circled squares in our figure).

\footnotetext{
${ }^{4}$ Note that PNe with different central star masses probe different epochs in the galactic history and also are subject to different selection effects in photometric surveys.
}

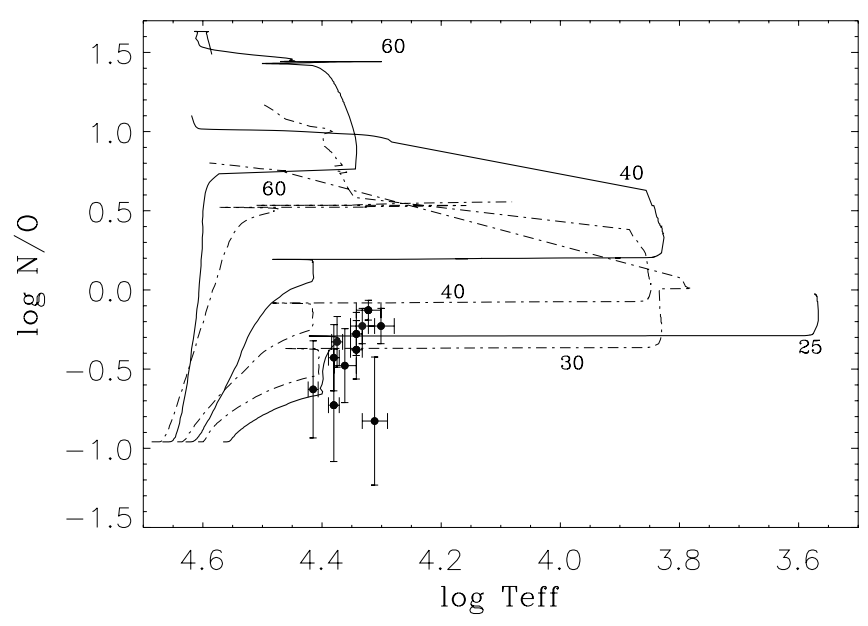

Fig. 14. - Surface N/O ratio (by number) against the effective temperature. Theoretical predictions as in previous figures. 


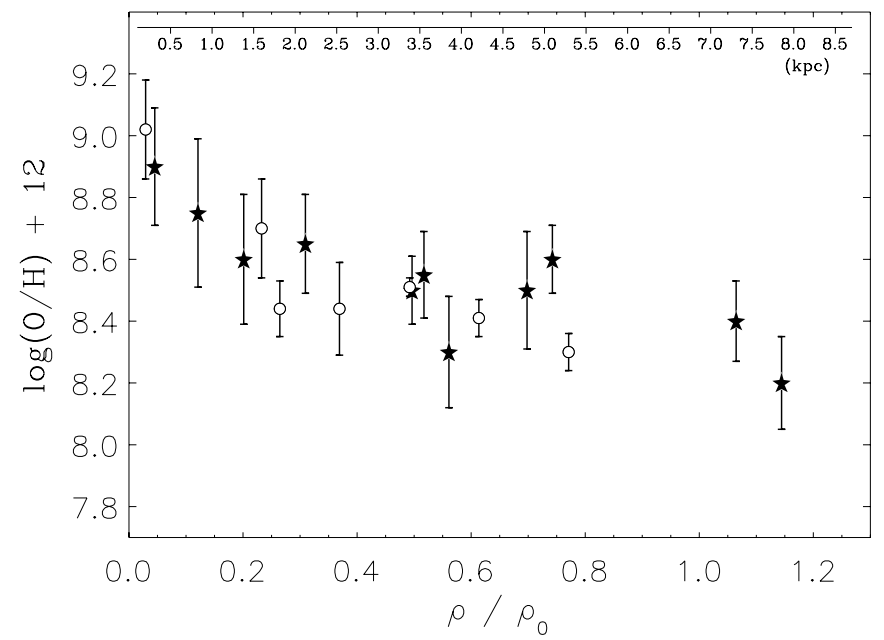

FIG. 15.-Radial distribution of $\mathrm{O}$ abundances. Open circles correspond to the $\mathrm{H}$ il region results from Vílchez et al. (1988), and filled stars stand for our B-type supergiant abundances. Note that the $x$-axis represents the deprojected distance to the galactic center, in units of the Holmberg radius $\left(\rho_{0}=28\right.$ ' $^{\prime} 77$, P.A. $=22^{\circ}$, and $i=54^{\circ}$ ). The upper $x$-axis shows the distances in units of kpc, while considering a distance to M33 of $820 \mathrm{kpc}$ (Freedman et al. 2001).

Figure 17 presents the comparison of the radial distribution of stellar ( filled stars) and ISM $\left(T_{e}\right) \mathrm{N}$ abundances from Vílchez et al. (1988; open circles). Although there is a relic of an initial $\mathrm{N} / \mathrm{H}$ radial gradient $\left(-0.018 \pm 0.005 \mathrm{dex} \operatorname{arcmin}^{-1}\right)$, the comparison to $\operatorname{ISM}\left(T_{e}\right)$ abundances nicely shows the effects of the chemical evolution on the stellar abundances (a mean difference of almost 0.7 dex) caused by internal mixing with $\mathrm{CN}$-cycled material. Note that it is generally accepted that $\mathrm{N}$ is one of the least depleted elements in interstellar gas (i.e., depletion onto dust grains); thus, this difference must be a consequence of the stellar evolution. It is tempting to interpret the difference in the stellar and ISM N/H slopes (see Fig. 18) in terms of "the lower the initial metallicity is, the larger the surface $\mathrm{N}$ enrichment results due to evolution." However, this needs to be investigated more carefully because of the presence of different initial masses and/or rotational velocities in our sample.

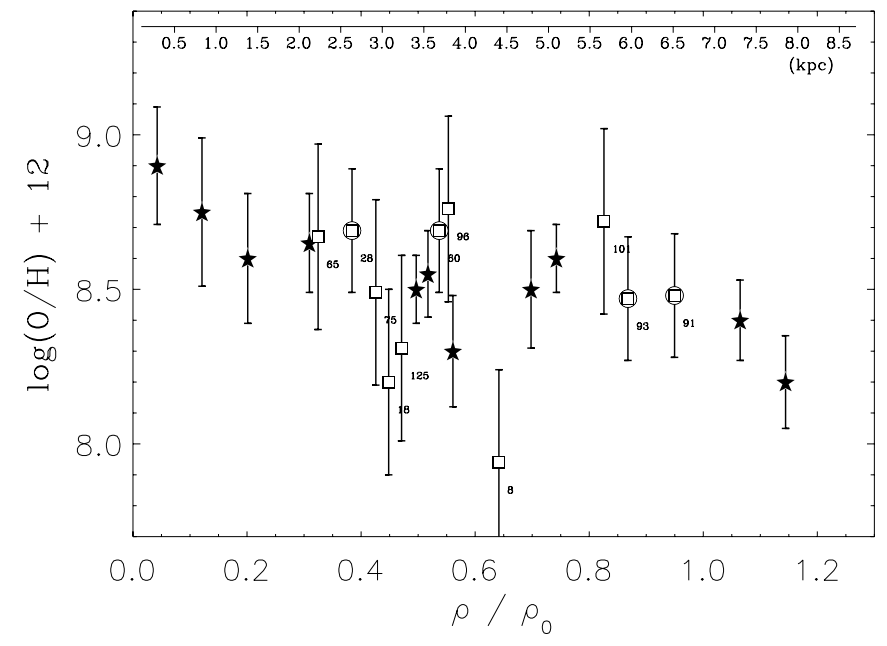

FIG. 16.-Comparison of the radial distribution of $\mathrm{O}$ abundances obtained from planetary nebulae (Magrini et al. 2004; open squares) and from our B-type supergiants ( filled stars). The agreement is good in general, in particular when considering the four PNe with well-established electron temperatures (represented by a circled square; see the above reference for a detailed discussion). The reader is referred to Fig. 15 for a description of the $x$-axis units.

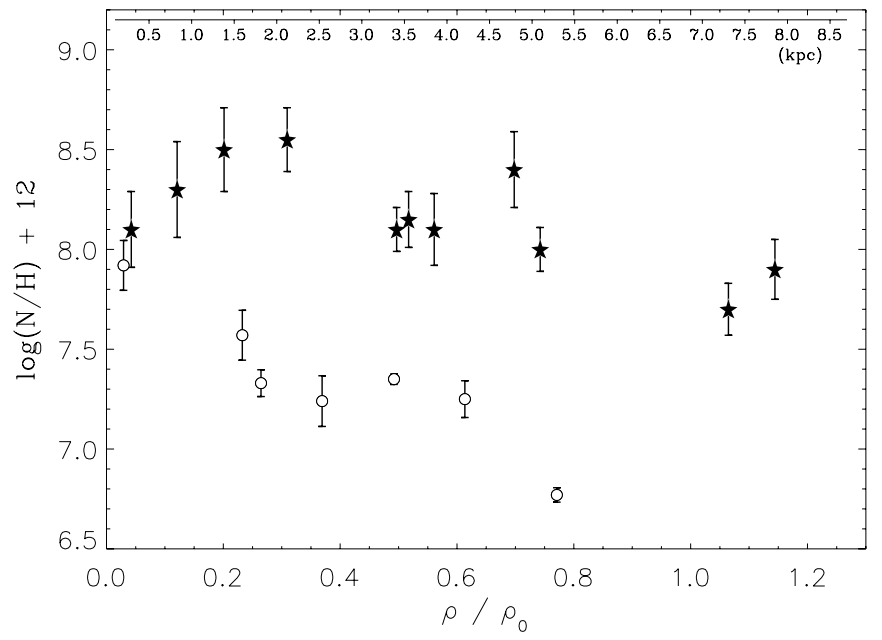

FIG. 17.- Radial distribution of $\mathrm{N}$ abundances. The open circles correspond to $\mathrm{H}$ II region results from Vílchez et al. (1988), based on direct determinations of the electronic temperature of the nebulae, while filled stars represent our B-type supergiants. Although a gradient is present in the stellar $\mathrm{N}$ abundances, the comparison with $\operatorname{ISM}\left(T_{e}\right)$ abundances shows the effects of the stellar evolution in the $\mathrm{N}$ surface abundances (around 1 dex of difference; see the text for a discussion). See previous figures for an explanation of the $x$-axis units.

Finally, we show in Figure 19 the radial abundance gradients obtained for the two other $\alpha$-elements, $\mathrm{Mg}$ (top panel) and $\mathrm{Si}$ (middle panel). The stellar abundances of both $\mathrm{Mg}$ and Si follow the $\mathrm{O}$ gradient, although showing smaller slopes (actually, the results for Si are also consistent with almost no gradient). For magnesium we find a radial gradient of $-0.010 \pm 0.004 \mathrm{dex} \operatorname{arcmin}^{-1}$ with a central $\mathrm{Mg}$ abundance of $7.60 \pm 0.07 \mathrm{dex}$ (note that B133 and UIT 122 have been excluded in the weighted least-squares fit). For Si the negative slope is $0.005 \pm 0.006 \mathrm{dex} \operatorname{arcmin}^{-1}$, and the central value is $7.52 \pm 0.11 \mathrm{dex}$. Based on the abundances of the three $\alpha$-elements $(\mathrm{O}, \mathrm{Mg}$, and $\mathrm{Si})$, we define a characteristic metallicity for each star as the mean of their individual differences with respect to the solar abundances. The bottom panel of Figure 19 displays the spatial behavior of this metallicity indicator (note that in contrast to the other panels in this figure, the $y$-axis is in linear units of $Z_{\odot}$ ). Thus, the B-type supergiants presented in this work cover a range in metallicity from $\sim 1.1$ to almost

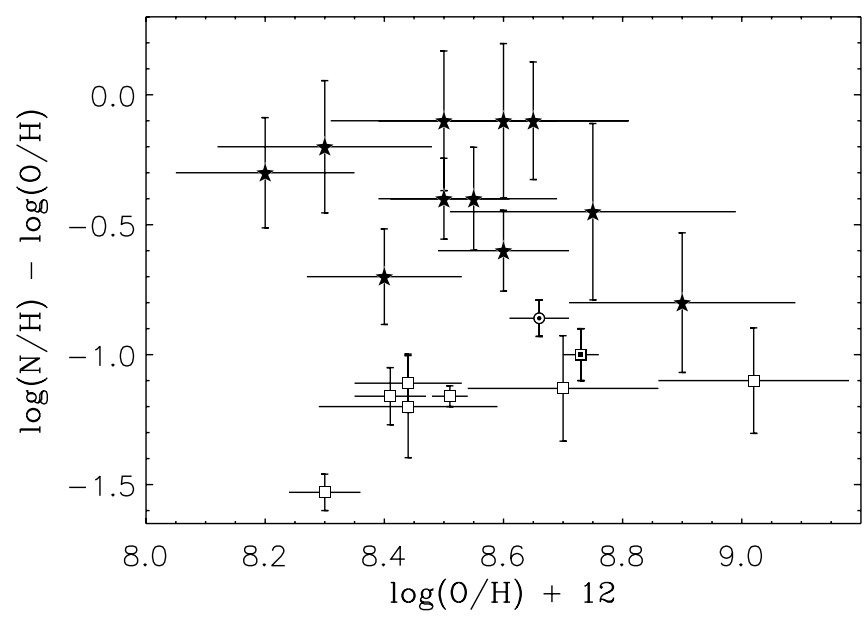

FIG. 18.-Comparison of N/O abundances obtained from the B-type supergiants (filled stars) and the $\operatorname{ISM}\left(T_{e}\right)$ results by Vílchez et al. (1988; open squares). We include in this diagram the location of the Sun and the recent revision of the abundance composition of the Orion Nebula M42 (partially filled square) by Esteban et al. (2004). 


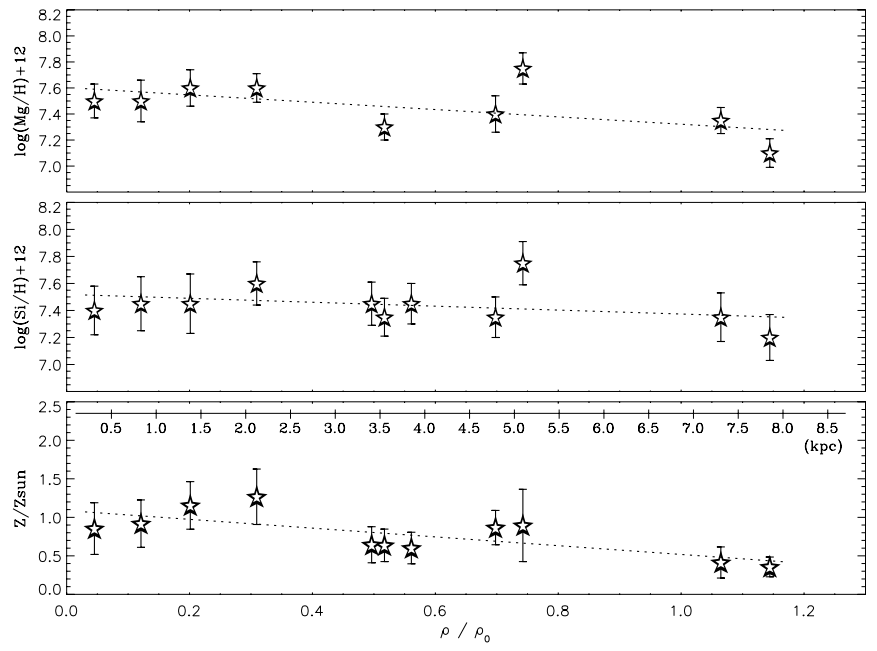

FIG. 19.- - Radial stellar abundance gradients of Mg (top), Si (middle), and the global metallicity indicator based on $\alpha$-element abundances (bottom). Weighted least-squares fits are also shown. Note that B133 and UIT 122 have been excluded in the Mg plot (see text for comments). The reader is referred to Fig. 15 for an explanation of the different $x$-axis and their units.

$0.4 Z_{\odot}$. A weighted least-squares fit results in a slope of $-0.020 \pm$ $0.006 Z_{\odot} \operatorname{arcmin}^{-1}$, with a central value of $1.09 \pm 0.12 Z_{\odot}$. Table 11 summarizes the different $\alpha$-element abundance gradients obtained in this work and several others taken from the available literature.

Vílchez et al. (1988) found a positive gradient in the ratio of $\mathrm{S}$ to $\mathrm{O}$ abundances. This is not expected from the point of view of stellar nucleosynthesis because both elements are supposed to have a common origin. Figure 20 displays the behavior of $\mathrm{S}$ (Vílchez et al. 1988) and stellar Mg and Si with respect to O abundances, showing a similar trend for the three $\alpha$-elements. While for each of the element ratios alone one might argue that small number statistics might have caused an artifact or that uncovered systematic effects might play a role, it is striking that the ISM S abundances and the stellar data, which are determined by a completely independent method, give a similar result. As a caveat, we note that our $\mathrm{Mg}$ abundances are based on one line only. On the

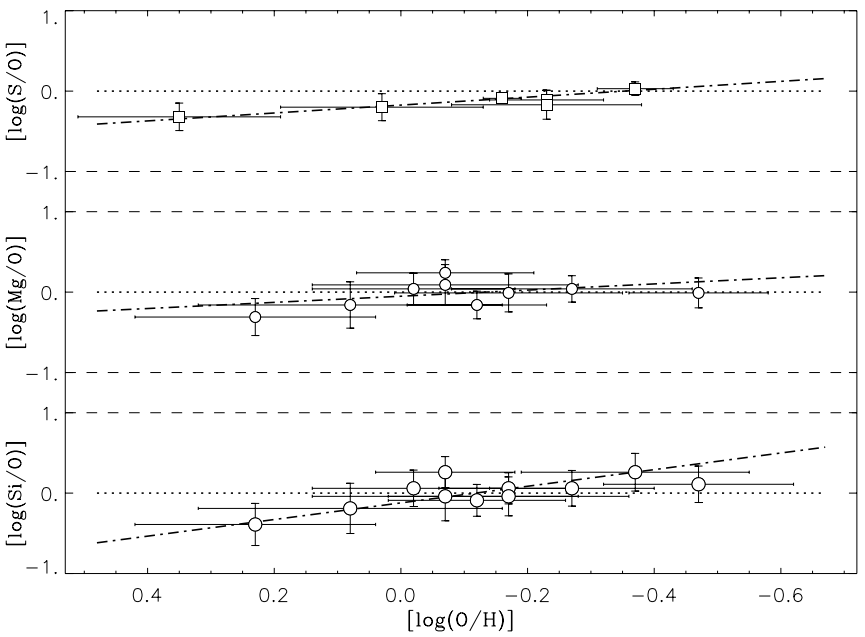

FIG. 20.-Dependence of the ratio of three different $\alpha$-elements to $\mathrm{O}$ abundances. S abundances (squares) are taken from H in regions (Vílchez et al. 1988), while $\mathrm{Mg}$ and $\mathrm{Si}$ abundances (circles) have been obtained from B-type supergiants. We have included the weighted least-squares fits (dot-dashed lines) and marked the loci of solar ratios (dotted line). The ratios, as well as the $\mathrm{O}$ abundances, refer to the solar values. Note that for Mg we have excluded B133, while we cannot derive the $\mathrm{Mg}$ abundance for UIT 122 (see text for comments); we use the notation $[\log (\mathrm{X} / \mathrm{Y})]=\log (\mathrm{X} / \mathrm{Y})-\log (\mathrm{X} / \mathrm{Y})_{\odot}$.

other hand, with the data for three different elements obtained by two independent methods this result is certainly worth a careful follow-up investigation with a larger and improved data set.

In their study of four B-type stars within $2.5 \mathrm{kpc}$ of Galactic center, Smartt et al. (2001b) found an opposite result, namely, that the abundances of $\mathrm{Mg}, \mathrm{S}$, and $\mathrm{Si}$ (among other elements) increase, although a flattening occurs for the $\mathrm{O}$ abundance gradient. However, this study may have suffered from selection effects as the objects were chosen due to their positions being slightly outside the disk and hence suffering low extinction. It is thus possible that they do not represent the true chemical composition of the inner Galactic disk (see, for example, the recent results obtained by Najarro et al. [2004] from the analysis of a sample of Wolf-Rayet stars close to the Galactic center). Our opposite result for M33 shows a flattening in $\mathrm{Mg}$ and $\mathrm{Si}$ (and $\mathrm{S}$ from $\mathrm{H}$ II

TABLE 11

Radial Abundance Gradients in M33

\begin{tabular}{|c|c|c|c|}
\hline Source & $\begin{array}{c}\text { Central Abundance } \\
\text { (dex) }\end{array}$ & $\begin{array}{l}\text { Gradient } \\
\left(\rho / \rho_{0}\right)\end{array}$ & $\begin{array}{c}\text { Gradient } \\
\left(\operatorname{dex} \operatorname{arcmin}^{-1}\right)\end{array}$ \\
\hline \multicolumn{4}{|c|}{ ISM } \\
\hline Vílchez et al. (1988), O/H ................. & $9.02 \pm 0.16^{\mathrm{a}}$ & $-0.48 \pm 0.21$ & $-0.017 \pm 0.007$ \\
\hline Garnett et al. (1997), O/H ................ & 8.88 & $\cdots$ & $-0.027 \pm 0.005$ \\
\hline \multicolumn{4}{|c|}{ BSGs } \\
\hline & This paper: & $-0.54 \pm 0.41$ & $-0.019 \pm 0.014$ \\
\hline 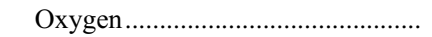 & $8.81 \pm 0.11$ & $-0.44 \pm 0.15$ & $-0.015 \pm 0.005$ \\
\hline 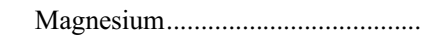 & $7.60 \pm 0.09$ & $-0.28 \pm 0.10$ & $-0.009 \pm 0.004$ \\
\hline 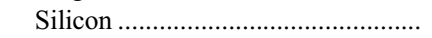 & $7.50 \pm 0.10$ & $-0.14 \pm 0.16$ & $-0.005 \pm 0.006$ \\
\hline 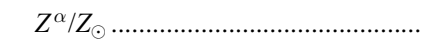 & $1.09 \pm 0.12^{\mathrm{b}}$ & $-0.57 \pm 0.18$ & $-0.020 \pm 0.006^{\mathrm{c}}$ \\
\hline
\end{tabular}

NoтE.- Note that unlike the other abundances, the entries relative to the metallicity, $Z^{\alpha}$, are represented in a linear scale.

a This abundance corresponds to the innermost $\mathrm{H}$ II region analyzed by these authors and not to the intersection obtained from a fit to the whole sample.

b This quantity is expressed in units of $Z_{\odot}$.

c This quantity is expressed in units of $Z_{\odot} \operatorname{arcmin}^{-1}$. 
regions) while $\mathrm{O}$ abundances increase. If both results are confirmed, M33 and the Galaxy would possibly have different star formation histories. The analysis of more supergiants close to the Galactic center, along with the inclusion of $\mathrm{S}$ in our spectral diagnostics, will allow us to further explore this question. Work is in progress in both fields.

\section{CONCLUSIONS}

Our analysis of M33 B-type supergiant stars confirms the existence of a (mild) radial dependence of the metallicity in the disk of the galaxy. The comparison of stellar and $\operatorname{ISM}\left(T_{e}\right)$ oxygen abundances shows good agreement within the uncertainties of the analysis and yields consistent radial abundance gradients for O. Moreover, there is independent evidence from the ISM and stellar data that the gradients in S (ISM) and Mg and Si (stellar) are shallower. Recent results for ISM Ne abundances presented by Willner \& Nelson-Patel (2002) support this surprising conclusion. For this $\alpha$-element the authors found a "best linear fit" 5 of $-0.008 \mathrm{dex} \operatorname{arcmin}^{-1}$ (or $-0.03 \mathrm{dex} \mathrm{kpc}-1$ once corrected for the difference in the adopted distance). We suggest the analysis of a larger sample of stars, in particular close to the Galactic center, to further investigate whether this detection is real. Should this be the case, it would provide a challenge to the present framework of nucleosynthesis and chemical evolution.

We found good agreement between PN and stellar O abundances. According to Magrini et al. (2004), no single PN in their sample is of type I, which means that the initial masses of their progenitors were below $\sim 2.4 M_{\odot}$, and then these progenitors were born $\sim 3$ Gyr ago (Stasińska 2004). Globally, the oxygen abundance is expected to be little affected by nucleosynthesis in the PN progenitors, in particular for such intermediate- to low-mass stars (Marigo 2001). Thus, if the fairly good agreement found between $\mathrm{PN}$ and B-type supergiant $\mathrm{O}$ abundances is confirmed in future studies, the conclusion is that the disk of M33 has not suffered a significant enrichment (at least in $\mathrm{O}$ ) in the last $3 \mathrm{Gyr}$. The same is found for the disk of the Milky Way (Maciel \& Quireza 1999).

Finally, we are ready to discuss the important issue of the metallicity dependence of the number ratio of WN to WC stars in nearby galaxies. With the results from this study, our previous work on NGC 300 (Urbaneja et al. 2005), and recent work on stellar abundances by other authors (SMC, Korn et al. 2000; Venn et al. 2000; Trundle et al. 2004; Trundle \& Lennon 2005; LMC, Korn et al. 2000; NGC 6822, Venn et al. 2002), we are able to base this solely on abundances obtained from stellar spectroscopy. Figure 21 shows the corresponding relationship. Note that we include only those galaxies for which a solid determination of their $\mathrm{O}$ abundances has been obtained on the basis of stellar spectroscopy. The WN/WC number ratios have been taken from the recent review by Massey (2003), except for NGC 300, taken from Schild et al. (2003); we note that they regard their value as a lower limit. The stellar oxygen abundances are taken from the references above, except for the three points describing the disk

\footnotetext{
5 It has to be noted that Willner \& Nelson-Patel (2002) prefer a two-value representation for their $\mathrm{H}$ il region $\mathrm{Ne}$ abundances; see the previous reference for further details.
}

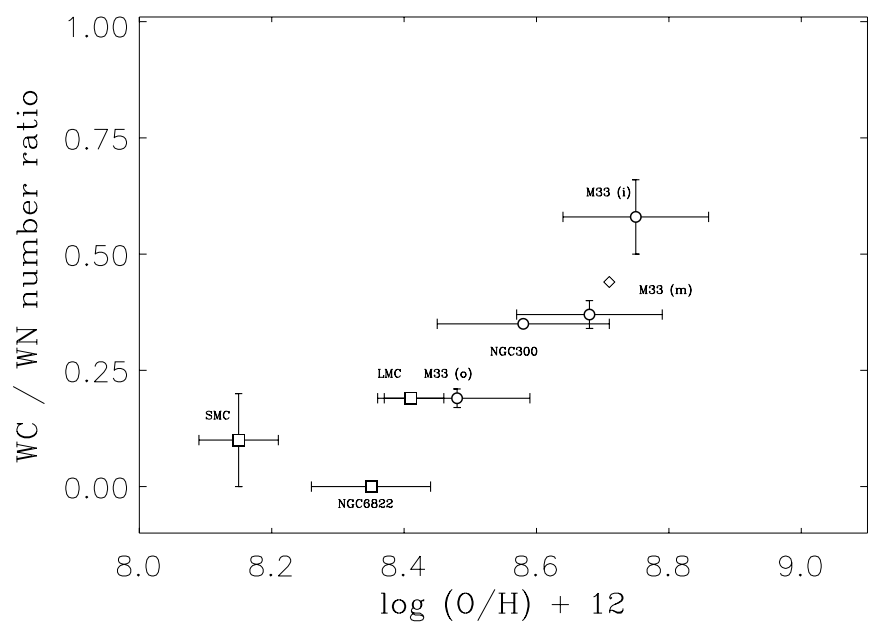

FIG. 21.-Observed number ratio of $\mathrm{WC}$ to $\mathrm{WN}$ in nearby galaxies with $\mathrm{O}$ abundances derived from early-type stars. The WC/WN number ratios have been taken from Massey (2003), except for NGC 300, taken from Schild et al. (2003). Oxygen abundance results of this work are used for the three points (inner, middle, and outer region) describing M33. The open diamond locates the position of M33 when considering only Wolf-Rayet stars with normalized galactocentric distance $\rho / \rho_{0} \leq 0.45$ (see text for a complete explanation).

of M33 (labeled in the figure as $i, m$, and $o$ ), which make use of our $\mathrm{O}$ abundance gradient. Stellar oxygen abundances, based on a completely different and consistent methodology, reproduce the results previously found by Massey \& Johnson (1998) on the basis of ISM O abundance, at least for the subsample of irregular and late-type spiral galaxies considered here. We consider this as a very satisfactory result, which provides a very useful constraint for stellar evolution theory (for a detailed discussion of the interplay of metallicity and the production of Wolf-Rayet stars, the reader is referred to Meynet \& Maeder 2005). Additional efforts, currently under way, are required to precisely locate the large spiral galaxies (Milky Way and M31) in this plot. The open diamond indicates the location of M33 when considering only WolfRayet stars with normalized galactocentric distances $\rho / \rho_{0} \leq 0.45$, to reproduce the spatial coverage observed in NGC 300 by Schild et al. (2003). These two galaxies, which have very similar structural parameters, illustrate a nice relationship, in which the more massive galaxy (M33) has the higher metallicity (O abundance), harboring at the same time a larger number of Wolf-Rayet stars and a higher ratio of $\mathrm{WC}$ to $\mathrm{WN}$.

The authors wish to recognize and acknowledge the very significant cultural role and reverence that the summit of Mauna Kea has always had within the indigenous Hawaiian community. We are most fortunate to have the opportunity to conduct observations from this mountain.

This work has been partly supported by Spanish MEC under projects PNAYA 2001-0436 and PNAYA 2004-08271-C02-02. F. N. also acknowledges grant PNAYA 2003-02785-E and the Ramón y Cajal program. The constructive and helpful remarks of an anonymous referee are gratefully acknowledged.
Allende Prieto, C., Lambert, D. L., \& Asplund, M. 2001, ApJ, 556, L63 Becker, S. R., \& Butler, K. 1988, A\&A, 201, 232

1989, A\&A, 209, 244

. 1990, A\&A, 235, 326

Bresolin, F., Gieren, W., Kudritzki, R.-P., Pietrzyński, G., \& Przybilla, N. 2002, ApJ, 567, 277

\section{REFERENCES}

Bresolin, F., Kudritzki, R.-P., Méndez, R. H., \& Przybilla, N. 2001, ApJ, 548, L159

Butler, K. 1984, Ph.D. thesis, Univ. College London

Butler, K., \& Giddings, J. 1985, Newsletter on Analysis of Astronomical Spectra, Vol. 9 (Univ. London)

Deharveng, L., Peña, M., Caplan, J., \& Costero, R. 2000, MNRAS, 311, 329

Eber, F. 1987, Diplomarbeit (Munich: Ludwig-Maximillians-Univ.) 
Eber, F., \& Butler, K. 1988, A\&A, 202, 153

Esteban, C., Peimbert, M., García-Rojas, J., Ruíz, M. T., Peimbert, A., \& Rodríguez, M. 2004, MNRAS, 355, 229

Fich, M., \& Silkey, M. 1991, ApJ, 366, 107

Freedman, W. L., et al. 2001, ApJ, 553, 47

Gabler, R., Gabler, A., Kudritzki, R. P., \& Pauldrach, A. W. A. 1989, A\&A, 226, 162

Garmany, C. D., \& Stencel, R. E. 1992, A\&AS, 94, 211

Garnett, D. R., Kennicutt, R. C., Jr., \& Bresolin, F. 2004, ApJ, 607, L21

Garnett, D. R., Shields, G. A., Skillman, E. D., Sagan, S. P., \& Dufour, R. J. 1997, ApJ, 489, 63

Gray, D. F. 1982, The Observations and Analysis of Stellar Photospheres (New York: Wiley)

Grevesse, N., \& Sauval, A. J. 1998, Space Sci. Rev., 85, 161

Haser, S. M. 1995, Ph.D. thesis, Univ. Munich

Heger, A., \& Langer, N. 2000, ApJ, 544, 1016

Heger, A., Langer, N., \& Woosley, S. E. 2000, ApJ, 528, 368

Herrero, A., Lennon, D. J., Vílchez, J. M., Kudritzki, R. P., \& Humphreys, R. H. 1994, A\&A, 287, 885

Herrero, A., Puls, J., \& Najarro, F. 2002, A\&A, 396, 949

Humphreys, R. M., \& Davidson, K. 1979, ApJ, 232, 409

Humphreys, R. M., \& Sandage, A. 1980, ApJS, 44, 319

Ivanov, G. R., Freedman, W. L., \& Madore, B. F. 1993, ApJS, 89, 85

Jokuthy, A. 2002, Diploma thesis, Univ. Munich

Kaufer, A., Venn, K. A., Tolstoy, E., Pinte, C., \& Kudritzki, R. P. 2004, AJ, 127,2723

Kennicutt, R. C., Jr., Bresolin, F., \& Garnett, D. R. 2003, ApJ, 591, 801

Korn, A., Becker, S. R., Gummersbach, C. A., \& Wolf, B. 2000, A\&A, 353, 655

Kudritzki, R. P. 1980, A\&A, 85, 174

Kudritzki, R.-P., \& Puls, J. 2000, ARA\&A, 38, 613

Kudritzki, R.-P., Puls, J., Lennon, D. J., Venn, K. A., Reetz, J., Najarro, F., McCarthy, J. K., \& Herrero, A. 1999, A\&A, 350, 970

Lamers, H. J. G. L. M., Nota, A., Panagia, N., Smith, L. J., \& Langer, N. 2001, ApJ, 551, 764

Lennon, D. J. 1997, A\&A, 317, 871

Lennon, D. J., Becker, S. T., Butler, K., Eber, F., Groth, H. G., Kunze, D., \& Kudritzki, R. P. 1991, A\&A, 252, 498

Lennon, D. J., Dufton, P. L., \& Cowley, C. 2003, A\&A, 398, 455

Lennon, D. J., Dufton, P. L., \& Fitzsimmons, A. 1992, A\&AS, 94, 569

Lyubimkov, L. S., Rostopchin, S. I., Rachkovskaya, T. M., Poklad, D. B., \& Lambert, D. L. 2005, MNRAS, 358, 193

Maciel, W. J., \& Quireza, C. 1999, A\&A, 345, 629

Maeder, A., \& Meynet, G. 2001, A\&A, 373, 555

Magrini, L., Cardwell, A., Corradi, R. L. M., Mampaso, A., \& Perinotto, M. 2001, A\&A, 367, 498

Magrini, L., Perinotto, M., Mampaso, A., \& Corradi, R. L. M. 2004, A\&A, 426, 779

Marigo, P. 2001, A\&A, 370, 194

Massey, P. 2003, ARA\&A, 41, 15

Massey, P., Armandroff, T. E., Pyke, R., Patel, K., \& Wilson, C. D. 1995, AJ, 110,2715

Massey, P., Bianchi, L., Hutchings, J. B., \& Stecher, T. P. 1996, ApJ, 469, 629

Massey, P., Hodge, P. W., Holmes, S., Jacoby, J., King, N. L., Olsen, K., Saha, A., \& Smith, C. 2001, BAAS, 33, 1496

Massey, P., \& Johnson, O. 1998, ApJ, 505, 793

McCarthy, J. K., Kudritzki, R. P., Lennon, D. J., Venn, K. A., \& Puls, J. 1997, ApJ, 482, 757

McCarthy, J. K., Lennon, D. J., Venn, K. A., Kudritzki, R. P., Puls, J., \& Najarro, F. 1995, ApJ, 455, L135

McErlean, N. D., Lennon, D. J., \& Dufton, P. L. 1998, A\&A, 329, 613

. 1999, A\&A, 349, 553

McWilliam, A. 1997, ARA\&A, 35, 503

Meynet, G., \& Maeder, A. 2003, A\&A, 404, 975

. 2005, A\&A, 429, 581

Mihalas, D., Hummer, D. G., \& Conti, P. S. 1972, ApJ, 175, L99

Monteverde, M. I., Herrero, A., \& Lennon, D. J. 2000, ApJ, 545, 813
Monteverde, M. I., Herrero, A., Lennon, D. J., \& Kudritzki, R. P. 1996, A\&A, 312,24

1997, ApJ, 474, L107

Najarro, F., Figer, D. F., Hillier, J. D., \& Kudritzki, R.-P. 2004, ApJ, 611, L105

Peimbert, M. 1967, ApJ, 150, 825

Pilyugin, L. S. 2003, A\&A, 399, 1003

Przybilla, N. 2002, Ph.D. thesis, Univ. Munich

Puls, J., Repolust, T., Hoffmann, T. L., Jokuthy, A., \& Venero, R. O. J. 2003, in IAU Symp. 212, A Massive Star Odyssey: From Main Sequence to Supernova, ed. K. van der Hucht, A. Herrero, \& C. Esteban (San Francisco: ASP), 61

Puls, J., et al. 1996, A\&A, 305, 171

2005, A\&A, 435, 669

Repolust, T., Puls, J., Hanson, M. M., Kudritzki, R.-P., \& Mokiem, M. R. 2005, A\&A, 440, 261

Repolust, T., Puls, J., \& Herrero, A. 2004, A\&A, 415, 349

Rudolph, A. L., Simpsom, J. P., Haas, M. R., Erikson, E., \& Fich, M. 1997, ApJ, 489, 94

Ryans, R. S. I., Dufton, P. L., Rolleston, W. R. J., Lennon, D. J., Keenan, F. P., Smoker, J. V., \& Lambert, D. L. 2002, MNRAS, 336, 577

Santolaya-Rey, E., Puls, J., \& Herrero, A. 1997, A\&A, 323, 488

Schild, H. Crowther, P. A., Abbott, J. B., \& Schmutz, W. 2003, A\&A, 397, 859

Sheinis, A. I., Bolte, M., Epps, H. W., Kibrick, R. I., Miller, J. S., Radovan, M. V., Bigelow, B. C., \& Sutin, B. M. 2002, PASP, 114, 851

Shetrone, M., Venn, K. A., Tolstoy, E., Primas, F., Hill, V., \& Kaufer, A. 2003, AJ, 125, 684

Smartt, S. J., Crowther, P. A., Dufton, P. L., Lennon, D. J., Kudritzki, R. P., Herrero, A., McCarthy, J. K., \& Bresolin, F. 2001a, MNRAS, 325, 257

Smartt, S. J., Lennon, D. J., Kudrityzki, R.-P., Rosales, F., Ryans, R. S. I., \& Wright, N. 2002, A\&A, 391, 979

Smartt, S. J., Venn, K. A., Dufton, P. L., Lennon, D. J., Rolleston, W. R. J., \& Keenan, F. P. 2001b, A\&A, 367, 86

Stasińska, G. 2002, Rev. Mex. AA Ser. Conf., 12, 62

. 2004, in Cosmochemistry, ed. C. Esteban et al. (Cambridge: Cambridge Univ. Press), 115

2005, A\&A, 434, 507

Tolstoy, E., Venn, K. A., Shetrone, M., Primas, F., Hill, V., Kaufer, A., \& Szeifert, T. 2003, AJ, 125, 707

Trundle, C., Dufton, P. L., Lennon, D. J., Smartt, S. J., \& Urbaneja, M. A. 2002, A\&A, 395, 519

Trundle, C., \& Lennon, D. J. 2005, A\&A, 434, 677

Trundle, C., Lennon, D. J., Puls, J., \& Dufton, P. L. 2004, A\&A, 417, 217

Urbaneja, M. A. 2004, Ph.D. thesis, Univ. La Laguna

Urbaneja, M. A., Herrero, A., Bresolin, F., Kudritzki, R.-P., Gieren, W., \& Puls, J. 2003, ApJ, 584, L73

Urbaneja, M. A., Herrero, A., Kudritzki, R. P., Bresolin, F., Corral, L. J., \& Puls, J. 2002, A\&A, 386, 1019

Urbaneja, M. A., et al. 2005, ApJ, 622, 862

Venn, K. A. 1995, ApJS, 99, 659 1999, ApJ, 518, 405

Venn, K. A., Brooks, A. M., Lambert, D. L., Lemke, M., Langer, N., Lennon, D. J., \& Keenan, F. P. 2002, ApJ, 565, 571

Venn, K. A., McCarthy, J. K., Lennon, D. J., Przybilla, N., Kudritzki, R. P., \& Lemke, M. 2000, ApJ, 541, 610

Venn, K. A., Tolstoy, E., Kaufer, A., Skillman, E. D., Clarkson, S. M., Smartt, S. J., Lennon, D. J., \& Kudritzki, R. P. 2003, AJ, 126, 1326

Vila-Costas, M. B., \& Edmunds, M. G. 1992, MNRAS, 259, 121

Vílchez, J. M., \& Esteban, C. 1996, MNRAS, 280, 720

Vílchez, J. M., Pagel, B. E. J., Díaz, A. I., Terlevich, E., \& Edmunds, M. G. 1988, MNRAS, 235, 633

Villamariz, M. R., Herrero, A., Becker, S. R., \& Butler, K. 2002, A\&A, 388, 940

Vrancken, M., Lennon, D. J., Dufton, P. L., \& Lambert, D. L. 2000, A\&A, 358, 639

Walborn, N. R., \& Fitzpatrick, E. L. 1990, PASP, 102, 379

Willner, S. P., \& Nelson-Patel, K. 2002, ApJ, 568, 679 